\title{
A model for cyclotron resonance scattering features
}

\author{
G. Schönherr ${ }^{1,2,3}$, J. Wilms ${ }^{2}$, P. Kretschmar ${ }^{3}$, I. Kreykenbohm ${ }^{1,4}$, A. Santangelo ${ }^{1}$, \\ R. E. Rothschild ${ }^{5}$, W. Coburn ${ }^{6}$, and R. Staubert ${ }^{1}$
}

1 Institut für Astronomie und Astrophysik, Abteilung Astronomie, Sand 1, 72076 Tübingen, Germany e-mail: gschoen@astro.uni-tuebingen.de

2 Dr. Karl Remeis-Sternwarte Bamberg, Astronomisches Institut der Friedrich-Alexander-Universität Erlangen-Nürnberg, Sternwartstr. 7, 96049 Bamberg, Germany

3 European Space Astronomy Centre, ESA, Apartado 50727, 28080 Madrid, Spain

4 INTEGRAL Science Data Centre, 16 Ch. d'Écogia, 1290 Versoix, Switzerland

5 Center for Astrophysics and Space Sciences, University of California, San Diego, CA 92093-0424, USA

6 Space Sciences Laboratory, University of California, Berkeley, CA 94720/7450, USA

Received 1 February 2007 / Accepted 27 June 2007

ABSTRACT

\begin{abstract}
Aims. We study the physics of cyclotron line formation in the high-energy spectra of accreting X-ray pulsars. In particular, we link numerical predictions for the line profiles to results from observational data analysis. Therefore, first we investigate the theoretical predictions and the significance of our model parameters, and second we aim at the development of a model to fit cyclotron lines in observational data.

Methods. Simulations were performed using Monte Carlo methods. The data were extracted with HEADAS 6.1.1 and INTEGRAL OSA 5.1. A convolution model for the cyclotron line shapes was implemented for the XSPEC spectral analysis software package and for data packages compatible with XSPEC local models.

Results. We predict the shapes of cyclotron lines for different prescribed physical settings. The calculations assume that the line-forming region is a low-density electron plasma, which is of cylindrical or slab geometry and which is exposed to a uniform, sub-critical magnetic field. We investigate the dependence of the shape of the fundamental line on angle, geometry, optical depth and temperature. We also discuss variations of the line ratios for non-uniform magnetic fields. We have developed a new convolution and interpolation model to simulate line features regardless of any a priori assumed shape of the neutron star continuum. Fitting RXTE and INTEGRAL data of the accreting X-ray pulsar V0332+53 with this model gives a qualitative description of the data. Strong emission wings of the fundamental cyclotron feature as predicted by internally irradiated plasma geometries are in principle observable by todays instruments but are not formed in V0332+53, hinting at a bottom illuminated slab geometry for line formation.
\end{abstract}

Key words. X-rays: binaries - stars: neutron - accretion, accretion disks - magnetic fields - line: formation - methods: numerical

\section{Introduction}

Cyclotron Resonance Scattering Features (hereafter called CRSFs or simply "cyclotron lines"), first discovered in the spectrum of Her X-1 (Trümper et al. 1977, 1978), are observed as absorption lines in the spectra of highly magnetized accreting Xray pulsars (Heindl et al. 2004). Their scientific importance lies in providing the only direct method currently known for the determination of the magnetic field of a neutron star (e.g. Harding \& Lai 2006; Orlandini \& Fiume 2001). The line energy $E_{\mathrm{cyc}}$ of the fundamental CRSF is approximately related to the magnetic field strength of the star by the " $12-B-12$ rule"

$E_{\mathrm{cyc}} \approx 11.57 \mathrm{keV} \cdot B_{12}$,

where $B_{12}$ denotes the magnetic field in units of $10^{12}$ Gauss (Canuto \& Ventura 1977). Due to the gravitational redshift, $z$, the observed line energy is shifted by a factor of $1 /(1+z)$ with respect to $E_{\mathrm{cyc}}$. Today, with the access to data from satellites as BeppoSAX, the Rossi X-ray Timing Explorer (RXTE), the International Astrophysics Gamma-Ray Observatory (INTEGRAL), and Suzaku, the diagnostic potential of CRSFs has grown anew: with these instruments the observed cyclotron line shapes are energetically resolved in detail for several sources, thus demanding an in-depth study of their formation. A better understanding of their complex line shapes would reveal much about the physical setting which drives the fascinating but up to today only poorly understood processes of accretion in X-ray pulsars. At present, the observed lines are usually modeled by Gaussian or Lorentzian shapes (Mihara et al. 1990; Makishima et al. 1990b). For modeling the fundamental feature, one must sometimes use several Lorentzians or Gaussians to obtain an acceptable fit of its non-trivial shape (Kreykenbohm et al. 2005).

In this paper, using Monte Carlo simulations based on an improved version of the code of Araya \& Harding (1999) and Araya-Góchez \& Harding (2000), we calculate line features for typical neutron star spectra and infer the line profiles under the assumption of physical parameters such as the accretion geometry, the viewing angle, and the plasma temperature. Based on our simulations, we develop a new local XSPEC model, cyclomc, for cyclotron lines and show first results from fitting observational data with cyclomc.

The outline of the present work is as follows: in Sect. 2 we summarize the basic theory of cyclotron line formation and describe standard scenarios for the accretion process onto the neutron star. We report key results from past observations and give an overview of different numerical approaches to modeling cyclotron lines for neutron stars. Section 3 focuses on the modeling approach taken here. We try to motivate our steps from the 
point of view of an observer, however, some rather technical discussion is necessarily included in that section. Theoretical predictions from Sect. 4 are complemented in Sect. 5 by a comparison of the model with real data. Finally, in Sect. 6 we summarize our results and discuss future steps for cyclotron line modeling.

\section{Overview}

\subsection{CRSF formation in X-ray pulsar spectra}

Cyclotron lines are found in the spectra of accreting neutron stars in binary systems with magnetic field strengths of the order $B \sim 10^{11}-10^{13}$ Gauss. See, e.g., Harding \& Lai (2006) for a review of the physics of strongly magnetized neutron stars. X-ray pulsars are thought to have masses of $M \sim 1.4 M_{\odot}$, radii $R \sim 10^{6} \mathrm{~cm}$, and luminosities of $10^{34}-10^{38} \mathrm{erg} \mathrm{s}^{-1}$. They accrete matter from a strong stellar wind or by Roche lobe overflow, usually through an accretion disk (Ghosh \& Lamb 1978). The magnetic field of the neutron star disrupts the flow of matter at the Alfvén radius, where the magnetic field pressure equals the ram pressure of the flow, and the matter is funneled along the field lines onto the magnetic poles (Basko \& Sunyaev 1976), reaching free-fall velocities of $\sim 0.4 c$. When discussing processes of accretion in the following, we restrict ourselves to the fielddominated volume near the neutron star surface at the magnetic poles. Inverse Comptonization of soft photons in the decelerated plasma produces photons in the X-and gamma-ray regime. The emission characteristics of this radiation depend on the mass accretion rate, $\dot{M}$. For large $\dot{M}$, a shock front develops at some distance from the neutron star surface, which does not permit the upscattered photons to escape vertically from the accretion column, i.e., parallel to the $B$-field. As a result, a "fan beam" emission pattern forms (Fig. 1). As was first shown by Basko \& Sunyaev (1976), the critical luminosity for shock formation, $L^{*}$, is

$L^{*}=2.72 \times 10^{37}\left(\frac{\sigma_{\mathrm{T}}}{\sqrt{\sigma_{\|} \sigma_{\perp}}}\right)\left(\frac{r_{0}}{R}\right)\left(\frac{M}{M_{\odot}}\right) \mathrm{erg} \mathrm{s}^{-1}$,

where $r_{0}$ is the polar cap radius, $\sigma_{\mathrm{T}}$ is the Thomson scattering cross section and $\sigma_{\|}$and $\sigma_{\perp}$ are the energy averaged cross sections for the scattering of photons which propagate in parallel and perpendicular to the magnetic field direction (Becker 1998). For small $\dot{M}$, on the other hand, i.e., for $L<L^{*}$, the radiation is emitted from an accretion mound such that most photons are emitted parallel to the $B$-field in a "pencil beam" pattern.

Before emerging from the line-forming region, the highenergy photons undergo scattering processes with the electrons in the relativistic plasma of the accretion column. The scattering cross section is resonant at energies determined by the separation of the Landau energies, the discrete energy levels of the electrons: when the strength of the magnetic field $B$ approaches the critical field strength, $B_{\text {crit }}=\left(m^{2} c^{3}\right) /(e \hbar)=44.14 \times 10^{12} \mathrm{G}$, the de Broglie radius of a plasma electron becomes comparable to its Larmor radius. Quantum mechanical treatment of the electrons' motion perpendicular to the magnetic field lines (Mészáros 1992; Daugherty \& Harding 1986) reveals a quantization of the electrons' momenta $p_{\perp} /\left(m_{\mathrm{e}} c\right)=n\left(B / B_{\text {crit }}\right)$. This translates into discrete energy levels, where the fundamental Landau level is given by the $12-B-12$ rule (1) and the higher harmonics have $n$ times this energy. For photon-electron scattering, relativistic effects lead to a slightly anharmonic spacing of the resonant photon energies. Due to the large scattering cross section at the resonances and due to the quasi-harmonic spacing of the thermally broadened Landau levels, photons of
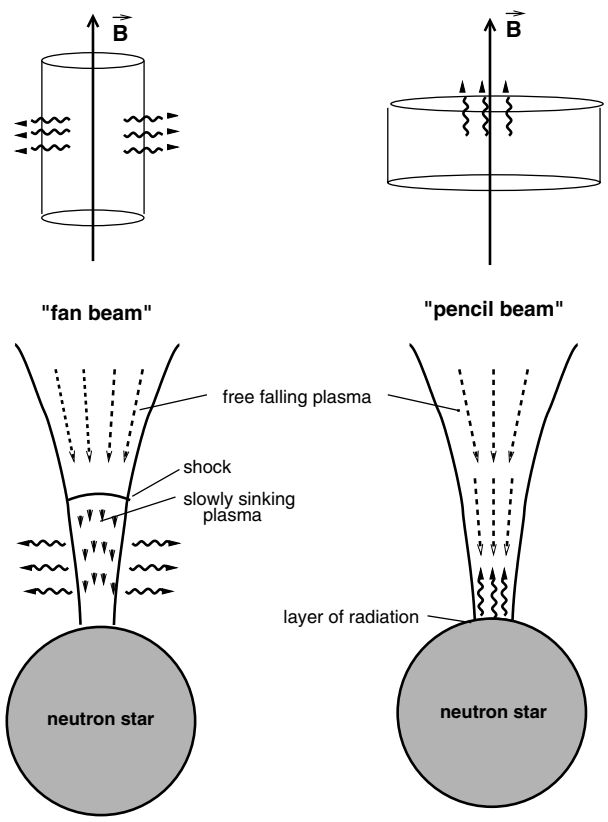

Fig. 1. Accretion geometries and radiation patterns. Left: "fan beam"/cylinder geometry. Right: "pencil beam"/slab geometry.

energies close to the Landau level energies may not escape the line-forming region unless inelastic scattering has slightly changed their energy. Consequently, absorption features in the photon spectrum are observed at

$E_{n}=m_{\mathrm{e}} c^{2} \frac{\sqrt{1+2 n\left(B / B_{\text {crit }}\right) \sin ^{2} \theta}-1}{\sin ^{2} \theta} \frac{1}{1+z}$,

where $m_{\mathrm{e}}$ is the electron rest mass, $c$ the speed of light, $\theta$ the angle between the photon direction and the magnetic field vector, and $z$ is the gravitational redshift at the radius of the line-forming region. Note that we enumerate the cyclotron lines starting at $n=1$, and refer to them as the first or fundamental line at the energy $E_{\mathrm{cyc}}=E_{1}$, followed by the second, third, fourth, etc. harmonics $(n=2,3,4, \ldots)$. The gravitational redshift at the neutron star surface is approximately ${ }^{1}$

$z=\frac{1}{\sqrt{1-\frac{2 G M}{R c^{2}}}}-1$

which gives $z \sim 0.3$ assuming the typical neutron star parameters given above. The thermal motion of the electrons parallel to the magnetic field lines remains free and is characterized by the parallel electron temperature, $T_{\mathrm{e}}$, which will be introduced in Sect. 3.3.

\subsection{Observations}

In 1976, the first cyclotron line was detected in the X-ray spectrum of Her X-1 (Trümper et al. 1977). First interpreted as an emission feature at $53 \mathrm{keV}$, the line was later proposed to be in absorption with theoretical arguments by Nagel (1981). Since the discovery of the Her X-1 cyclotron line, more sources exhibiting CRSFs have been observed (e.g., Heindl et al. 2004; Staubert 2003; Coburn et al. 2002; Santangelo et al. 2000).

\footnotetext{
1 Strictly spoken, Eq. (4) is exact only for a non-rotating, spherically symmetric, uncharged mass.
} 
At the time of writing, more than 16 accreting pulsars with securely detected cyclotron lines with surface magnetic fields in the range of $1-5 \times 10^{12} \mathrm{G}$ were known. The record holder with respect to the number of lines detected is $4 \mathrm{U} 0115+63$ (Heindl et al. 1999; Santangelo et al. 1999) where five features were found (Heindl et al. 2000).

CRSF sources are regular targets for observations. The progress over the last decades in energy resolution of instruments on satellites like BeppoSAX, RXTE, INTEGRAL, and Suzaku has led to excellent observational data of many interesting objects with complex cyclotron line features and has made high-quality phase resolved spectroscopy possible. As a result, many interesting characteristics of CRSFs are known today, awaiting a deeper explanation than given by the simple picture of line formation which was outlined in the previous section. Some key results from observational studies are:

1. The profile of the fundamental line is resolved, is clearly non-Gaussian, and exhibits a complex shape.

2. The second harmonic generally appears deeper than the fundamental line.

3. Significant variations of the line parameters of the CRSFs with the pulse phase are observed for some sources.

4. The line ratios are not necessarily harmonic. The deviations from the harmonic energies in some spectra are too large to be explained only by the basic relativistic corrections implied by Eq. (3).

5. The line position of the fundamental CRSF can vary with the source luminosity. Negative (Mihara 1995; Mowlavi et al. 2006; Nakajima et al. 2006; Tsygankov et al. 2006) and positive (La Barbera et al. 2005; Staubert et al. 2007) linear energy-to-luminosity correlations have been found.

\subsection{Numerical models}

There are two very different approaches to modeling the radiative transfer in the accretion column: solving finite difference equations and Monte Carlo simulations.

\subsubsection{Solving difference equations}

Motivated by the Her X-1 line detection, Nagel (1980, 1981) and later Mészáros \& Nagel (1985) employed Feautrier methods in order to solve the radiation transfer equation. They performed two sets of calculations, treating effects of anisotropy and Comptonization separately. Having first presented a lineformation mechanism for a cyclotron emission feature (Nagel 1980), in a later paper considering Comptonization effects, Nagel (1981) then favored the Her X-1 line to appear in absorption. For the combined effects of anisotropy and Comptonization Mészáros \& Nagel (1985) compared model predictions for different geometries (slab and cylinder geometry with internal or external illumination) and discussed variations with the angle of the emergent spectra. Their approach was later refined by the inclusion of higher harmonics (Alexander \& Mészáros 1991) or by including radiation pressure and temperature corrections in the atmosphere (Bulik et al. 1992, 1995). Recently, the influence of a non-uniform magnetic field in the line-forming region on the formation of CRSFs has been investigated with similar techniques by Nishimura, who found a variation of the line ratios of the CRSFs for the case of a dipolar (Nishimura 2003) and for a linearly varying (Nishimura 2005) magnetic field.

\subsubsection{Monte Carlo simulations}

Yahel (1979) was the first to use Monte Carlo simulations for simulating the CRSF formation in the atmosphere of a magnetized neutron star. He considered the formation of pulse profiles and X-ray spectra and found that the Her X-1 feature could indeed be reproduced as a consequence of resonant scatterings of extraordinary polarized photons. Two years later, Pravdo \& Bussard (1981) calculated angle-dependent pulsar spectra, including relativistic corrections to the Compton cross section and considering polarization dependence. Focusing on the continuum spectral shape they found a hardening of the spectra towards the magnetic equator. Wang et al. (1989b) performed Monte Carlo simulations for the geometry of a plane parallel slab with the slab normal parallel to the $B$-field vector, and the plasma being illuminated from below. This geometry was named "1-0 geometry" (Freeman et al. 1999; Isenberg et al. 1998b), in contrast to the "1-1 geometry" which indicates a homogeneous slab illuminated at the mid plane. While in the 1-0 geometry photons which return to the source plane after scattering are absorbed ("reflected photon flux"), in the 1-1 geometry photons may cross the source plane and the reflected and transmitted flux are symmetric. Results from a generalized model, where the slab normal may have any direction with respect to the $B$-field were discussed by Isenberg et al. (1998a,b). For the case where the slab normal is perpendicular to the magnetic field vector, their results are comparable to assuming a cylinder geometry for the lineforming region. Isenberg et al. (1998b) distinguished between line shapes of optically thin and optically thick matter. As one key result these authors found that the line wings disappear either for the 1-1 geometry and optically thick media or for the 1-0 geometry and optically thin media. However, none of these scenarios could explain the observed fundamental shallow and broad features due to the high equivalent width of the fundamental in both cases.

Inspired by the detection of up to two cyclotron lines ${ }^{2}$ at 50 and $100 \mathrm{keV}$ (Kendziorra et al. 1994) and at $110 \mathrm{keV}$ (Grove et al. 1995) during subsequent outbursts of the transient source A0535+26 in 1989 and 1994, Araya and Harding presented a new set of Monte Carlo simulations for very hard spectra of X-ray pulsars with near-critical fields (Araya \& Harding 1996, 1999; Araya-Góchez \& Harding 2000). For a low-density plasma and hence low continuum optical depths, they produced spectra for slab (1-1) and cylinder geometry of a plasma threaded by near-critical magnetic fields and discussed the influence of parameters as geometry, optical depth and anisotropy of the photon source on the line shapes. The results presented in this paper are based on their approach.

\section{Modeling CRSFs}

\subsection{Aims}

The key objective of this work is to obtain a physically motivated model for CRSF formation which is directly comparable to observational data. Firstly, such a comparison is fundamental when testing and reconsidering the validity of the model. Secondly, once the model has reached a well-developed state, only its simple applicability to real observational data provides the means for a systematic investigation of CRSF sources.

\footnotetext{
2 Recently it was shown that both lines claimed by Kendziorra et al. (1994) are present in the source spectrum (Kretschmar et al. 2005; Wilson \& Finger 2005; Inoue et al. 2005; Caballero et al. 2007).
} 


\subsection{Methods}

In order to achieve the desired flexibility, we base our model on Monte Carlo simulations using a revised, generalized version of the Araya \& Harding code. A new key feature is a Green's functions approach giving independence from any continuum model assumed. Araya \& Harding's implementation of an internally irradiated slab geometry is generalized to include also the case of illumination from the bottom (1-0 geometry). More information on the Monte Carlo implementation and details of the Green's function approach are given in Sect. 3.4. Due to the variety of sources and the uncertainty of the general physical picture, calculations are performed on a large multidimensional parameter grid. All simulation results are merged into archives in the form of FITS tables which are available from the authors. Line features for X-ray pulsar spectra for different physical settings (as outlined in the next section) within our parameter scope may be produced from these tables with a special convolution and interpolation model, also implemented as a local model for XSPEC (Arnaud 1996) and other analysis packages such as ISIS (Houck \& Denicola 2000).

\subsection{Physical setting}

We simulate the propagation of photons through a medium of prescribed physical conditions. The photons interact with the electrons in the medium via resonant scattering processes. The conditions in the line-forming region of the electron plasma are governed by the following parameters (Araya \& Harding 1999):

1. Magnetic field $[\boldsymbol{B}]$ As a first approximation we consider a neutron star with a magnetic field which is assumed to be uniform on the scale of the line-forming region. The field strengths simulated are between $1 \times 10^{12}$ and $7 \times 10^{12}$ Gauss, encompassing the whole range of $B$-fields found in cyclotron line sources. In Sect. 4.3 a possible generalization to nonuniform magnetic fields is described.

2. Plasma electrons $\left[T_{\mathrm{e}}, f\left(p_{\mathrm{e}}\right), \boldsymbol{n}\right]$ We consider a low-density thermal plasma. We also assume that all electrons are initially in their fundamental Landau state $n=0$. This assumption is justified by the very high cyclotron radiative decay rate for sub-critical fields

$r_{\text {rad }}=3 \times 10^{15} B_{12}^{2} \mathrm{~s}^{-1}$

compared to the collisional excitation rate

$r_{\text {col }}=5 \times 10^{8}\left(n_{\mathrm{e}} / 10^{21} \mathrm{~cm}^{-3}\right) B_{12}^{-3 / 2} \mathrm{~s}^{-1}$

(Latal 1986; Bonazzola et al. 1979). For their motion parallel to the $B$-field vector, we assume a thermal distribution of the electrons with their parallel momenta $p_{\mathrm{e}}$ given by a relativistic Maxwellian distribution

$f\left(p_{\mathrm{e}}\right) \mathrm{d} p_{\mathrm{e}} \propto \exp \left(-\frac{m_{\mathrm{e}} c^{2}\left(\sqrt{1+\left(\frac{p_{\mathrm{e}}}{m_{\mathrm{e}} c}\right)^{2}}-1\right)}{k T_{\mathrm{e}}}\right) \mathrm{d} p_{\mathrm{e}}$,

where $T_{\mathrm{e}}$ is the parallel electron temperature and $k$ is the Boltzmann constant. In the literature, $T_{\mathrm{e}}$ is often linked to the strength of the magnetic field (e.g. Lamb et al. 1990; Isenberg et al. 1998b; Araya \& Harding 1996, 1999; Araya-Góchez \& Harding 2000). We take these studies into account in order to determine the order of magnitude of the plasma temperature, but leave $T_{\mathrm{e}}$ as a free parameter in our

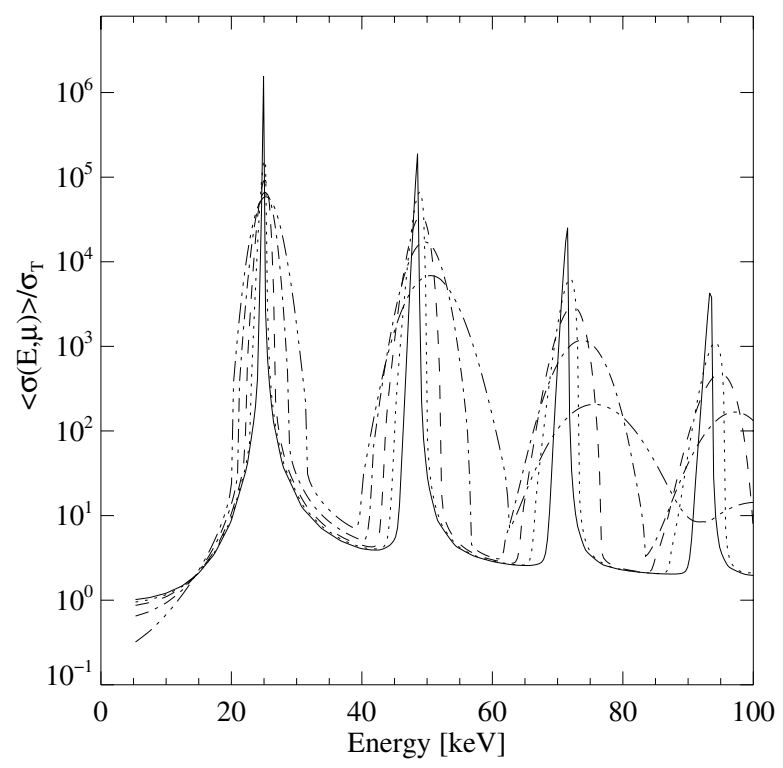

Fig. 2. Cross sections for $B / B_{\text {crit }}=0.05$ and $k T_{\mathrm{e}}=3 \mathrm{keV}$, thermally averaged over the sampled electron momenta, and in units of the Thompson cross section $\sigma_{\mathrm{T}}$. The resulting profiles are shown for different angles $\theta$ of the photon's direction with respect to the magnetic field vector. Solid lines: $\cos \theta=0.005$ and $\cos \theta=0.875$. Dotted, dashed-dotted and dashed lines (sharper towards broader): $\cos \theta=$ 0.200, 0.375, 0.625. Figure based on Araya \& Harding (1999).

simulations in order to keep the model's flexibility (see also Sect. 4.4). We also assume a slowly sinking plasma where bulk plasma motion may be neglected.

3. Optical depth $\left[\tau_{\mathrm{T}}\right]$ The Thomson optical depth $\tau_{\mathrm{T}}$ of the plasma is prescribed. The optical depth for cyclotron scattering $\tau_{\text {cyc }}$ relates to the Thomson optical depth as

$\tau_{\mathrm{cyc}}=\frac{\sigma_{\mathrm{cyc}}}{\sigma_{\mathrm{T}}} \tau_{\mathrm{T}}$,

implying a scattering optical depth $\tau_{\text {cyc }}$ which can be a factor of $\sim 10^{5}$ larger than $\tau_{\mathrm{T}}$ at the resonances of $\sigma_{\text {cyc }}$. Figure 2 shows the thermally averaged cross section $\left\langle\sigma_{\text {cyc }}\right\rangle / \sigma_{\mathrm{T}}$ as a function of energy and angle, calculated as a second order QED process (Sina 1996). Besides a highly resonant behavior of the cross section at the Landau energies, Fig. 2 also illustrates the angle-dependent relativistic shift in the resonances as well as the thermal broadening of the profiles. We calculate line features for Thomson optical depths between $\tau_{\mathrm{T}}=1 \times 10^{-4}$ and $\tau_{\mathrm{T}}=3 \times 10^{-3}$ (Araya \& Harding 1999). Depending on the plasma geometry, the resulting mean free path of a photon in the line-forming region is different for the same trajectory. The Thomson optical depths we simulate correspond to electron column densities $N_{\mathrm{e}}=\tau_{\mathrm{T}} / \sigma_{\mathrm{T}}$ between $1.5 \times 10^{20} \mathrm{~cm}^{-2}$ and $4.5 \times 10^{21} \mathrm{~cm}^{-2}$. These values are comparable to values assumed in other recent numerical or analytical studies. For instance, column densities of $N_{\mathrm{e}} \sim 10^{21}-10^{22} \mathrm{~cm}^{-2}$ are assumed by Nishimura (2003, 2005 ), and values of $N_{\mathrm{e}} \sim 10^{22} \mathrm{~cm}^{-2}$ are inferred by Becker \& Wolff (2007) for the sources Her X-1, LMC X-4, and Cen X-3.

4. Geometry [geo: sl/cy] We distinguish two basic geometries of the line-forming region motivated by the complementary "standard" pictures of accretion depicted in Fig. 1 (see also section 2.1 and Basko \& Sunyaev 1976): for the case of flow stopping through nuclear collisions at the 
surface, we adopt the geometry of a thin, plane-parallel slab (Mészáros et al. 1983; Harding et al. 1984). Radiative shocks or shocks from collisionless instabilities, on the other hand, require a cylindrical shape of the $\mathrm{X}$-ray emitting region (e.g., Becker \& Wolff 2007, and references therein). The heights of slab atmospheres are expected to be significantly smaller (Lamb et al. 1973; Wang et al. 1988) than typical radii, $h \ll r_{0}$, of the accretion mound (Ostriker \& Davidson 1973; Becker \& Wolff 2007). For cylindrical geometries, Becker \& Wolff (2007) investigated a more complicated radiative shock structure with a velocity gradient. For several X-ray pulsars, these authors found height to width ratios, $z_{\max } / r_{0}$, of the emitting region of the order $10^{2}$, confined to a volume smaller than the one of trapped radiation. Our simulations also suggest that practically all photons escape before reaching such boundaries, justifying our assumption of infinite extended plane-parallel line-forming regions. For the cylinder, we assume that the plasma is irradiated internally with the photon source located at the cylinder axis (Araya \& Harding 1999; Araya-Góchez \& Harding 2000). Two locations of the source plane are considered for the slab. The scenario of a line formation region above an isotropically emitting source is realized by a bottom-illuminated slab (Freeman et al. 1999; Isenberg et al. 1998b; Wang et al. 1989a), while placing the photon source at the midplane of a plane-parallel slab is representative of the scenario of line formation in an isothermal, semi-infinite atmosphere (Slater et al. 1982).

The optical depth the photons see into direction $\theta$ when covering an optical depth $\Delta \tau_{\mathrm{T}}$ along the slab normal or perpendicular to the cylinder depends on the geometry as:

$$
\begin{aligned}
& \Delta \tau_{\mathrm{T}}(\theta)=\frac{\Delta \tau_{\mathrm{T}}}{\sin \theta} \quad \text { (cylinder geometry) } \\
& \Delta \tau_{\mathrm{T}}(\theta)=\frac{\Delta \tau_{\mathrm{T}}}{\cos \theta} \quad(\text { slab geometry }) .
\end{aligned}
$$

\subsection{Technical realization}

We use a Monte Carlo method to simulate the resonant scattering processes between incident photons and plasma electrons which lead to the formation of cyclotron line features. The code used is a revised version of the code of Araya \& Harding (Araya \& Harding 1996, 1999; Araya-Góchez \& Harding 2000). The calculation of the relativistic cross sections is done with a separate code by Sina (1996). Resonant scattering with electrons up to the fourth harmonic is included.

Besides relaxed geometrical constraints on photon injection, and technical modifications such as an improved angular and energy resolution and increased statistics, the main difference of our program with respect to the preceeding one is the Green's functions approach: in each Monte Carlo run we insert 10000 photons of the same incident energy, $E_{\text {in }}$, pick for each photon a random angle $\theta_{\text {in }}\left(\cos \left(\theta_{\text {in }}\right) \in(-1,1)\right)$ with respect to the magnetic field direction, propagate them through the plasma (see below), and calculate from all final states the probabilities for photon redistribution into different energy and angular bins. The initial angular distribution of the photons is assumed to be isotropic. The relevant energy range $\left\{E_{\text {in }}\right\}$ for cyclotron line formation is assessed as follows: using the 12- $B$ 12 rule and assuming quasi-harmonic spacing of the cyclotron lines, the energy range containing the first four Landau levels can be fixed independently from the magnetic field strength in terms of $E / B_{12}$. Due to the link of resonant energy and magnetic field, the choice of this scale is important. First of all, it gives an optimized resolution of the CRSF features in the same way for different magnetic fields. Even more important, the choice of this scale is fundamental for later interpolation of the Green's functions: as the resonant energies are directly linked to the magnetic field, an interpolation of the line shapes in $E / B_{12}$-space ensures consistent results. For $E_{\text {in }} / B_{12} / \mathrm{keV} \in[6,48]$, we obtain a grid of Green's functions $G\left(E_{\text {in }} \rightarrow E_{\text {out }}, \theta_{\text {out }}\right) . E_{\text {in }}$ is sampled by 161 Monte Carlo runs, the resolution of the redistributed energies $E_{\text {out }}$ is given by an internal energy binning of 640 bins.

Each Monte Carlo photon is injected into the plasma with energy $E_{\text {in }}$ and direction $\theta_{\text {in }}$. The photon is then propagated according to its mean free path $1 /\left(n_{\mathrm{e}}\left\langle\sigma\left(E_{\text {in }}, \cos \theta_{\text {in }}\right)\right\rangle\right)$ and an electron is picked as a scattering partner. The electron is characterized by its parallel momentum, $p_{\mathrm{e}}$ (Eq. (7)), and its Landau state $n$. According to the scattering cross section (obtained from interpolation of previously calculated and tabulated values as a function of $B$ ), the state of the electron-photon pair changes from its incident configuration $\left(E^{(0)}, \theta^{(0)}\right)+\left(p_{\mathrm{e}}^{(0)}, n^{(0)}\right)$ to a different state $\left(E^{(1)}, \theta^{(1)}\right)+\left(p_{\mathrm{e}}^{(1)}, n^{(1)}\right)$. The new mean free path of the photon is calculated and the photon is propagated further. If the electron remains in an excited Landau state $n^{\prime}>0$ after scattering, another photon is emitted with $\left(E^{(2)}, \theta^{(2)}\right)+\left(p_{\mathrm{e}}^{(2)}, n^{(2)}\right)$ and processed further. This photon spawning can produce up to three secondary photons. Once a photon has escaped from the plasma, its contribution to the output spectrum is stored. Fixing the input angular distribution of the incident photons to be isotropic in the scope of the present work is done for reasons of simplicity, and in order to keep the computational expenses reasonable. In a future paper we will discuss the generalization to arbitrary angular photon distributions. Polarization of the photons is not included, however, polarized photons should yield a comparable picture (Wang et al. 1988) for the low-density regime chosen.

Calculations were performed in six-dimensional parameter space for a non-regular grid of points $\left[B / B_{\text {crit }}, T_{\mathrm{e}}, \mu, \tau_{\mathrm{T}}, E_{\mathrm{in}}\right.$, geo $]$ within the ranges $B / B_{\text {crit }} \in[0.03,0.15], k T_{\mathrm{e}} \in[2.5,20] \mathrm{keV}$, $\mu=\cos \left(\theta_{\text {out }}\right) \in(0,1), \tau_{\mathrm{T}} \in\left[1 \times 10^{-4}, 3 \times 10^{-3}\right], E_{\text {in }} / B_{12} / \mathrm{keV} \in$ $[6,48])$, and for slab $1-1$, slab $1-0$, and cylinder geometry respectively. The current parameter grid is resolved into $\left(N_{B / B_{\text {crit }}} \times\right.$ $\left.N_{T_{\mathrm{e}}} \times N_{\mu} \times N_{\tau_{\mathrm{T}}} \times N_{E_{\text {in }}} \times N_{\text {geo }}\right)=(16 \times 4 \times 8 \times 4 \times 161 \times$ 3) grid points requiring a simulation time of the order of $10^{5} \mathrm{CPU}$ hours on $2 \mathrm{GHz}$ workstations. The resolution was chosen such that the variation of the Green's functions between two points is sufficiently small to allow for interpolation. Hence, we can predict CRSFs by convolution of a continuum spectrum for any parameter combination $\left(B / B_{\text {crit }}, T_{\mathrm{e}}, \mu, \tau_{\mathrm{T}}\right.$, geo $)$ on this grid as follows: first, we obtain the corresponding Green's functions $G^{*}$ by linear interpolation in all parameters except the geometry on $E / B_{12}$. A set of Green's functions $\left\{G^{*}\left(E_{i} \rightarrow E_{j}, \theta_{k}\right)\right\}_{j}$ for a fixed example input energy $E_{i}$ and fixed physical setting is shown in Fig. 3. Second, we calculate the emergent photon flux $F^{\mathrm{em}}\left(E_{j}, \theta_{k}\right)$, i.e., the number of photons per $\mathrm{keV}$ in the $j$ th energy bin and $k$ th angular bin (binned in $\cos \theta$ ), as a function of the (isotropic) incident continuum flux $F^{\text {cont }}$ by convolving $F_{\text {cont }}$ with this interpolated set of Green's functions

$F^{\mathrm{em}}\left(E_{j}, \theta_{k}\right)=\frac{\sum_{i} G^{*}\left(E_{i} \rightarrow E_{j}, \theta_{k}\right) F^{\mathrm{cont}}\left(E_{i}\right) \Delta E_{i}}{\Delta E_{j}}$

Note that because of this approach CRSFs for arbitrary continuum shapes can be calculated without rerunning the simulations. 


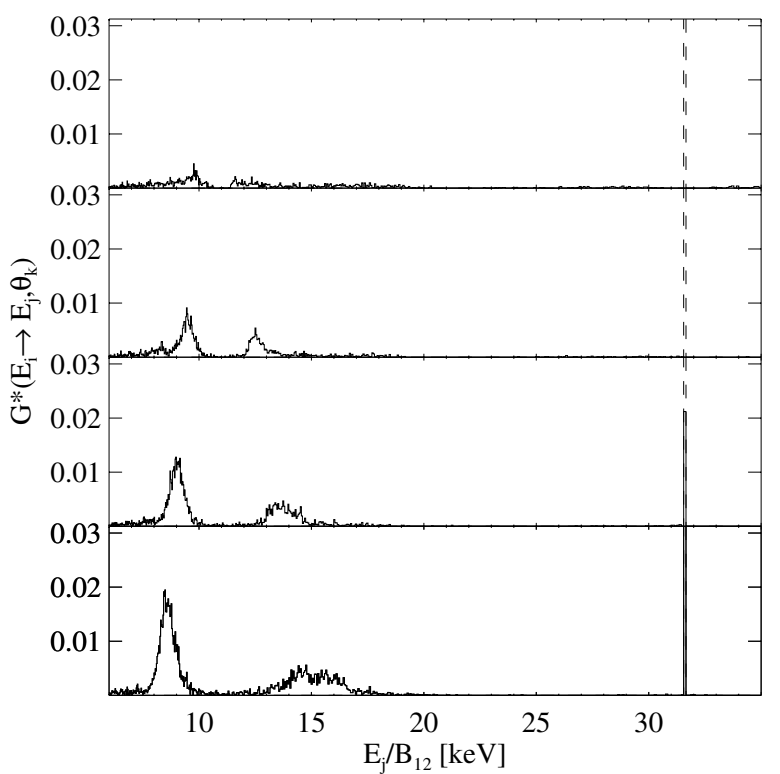

Fig. 3. Green's functions plotted for one input energy $E_{i} / B_{12} / \mathrm{keV}=$ 31.6 (dashed lines) against all output energies $\left\{E_{j}\right\}_{j}$, and into four (of eight) $\cos \theta$ bins ( $\cos \theta \in[0.125,0.250),[0.375,0.500),[0.625,0.750)$, $[0.875,1.0)$ from top to bottom). The physical setting is as follows: geo:sl, $B / B_{\text {crit }}=0.06, k T_{\mathrm{e}}=5 \mathrm{keV}, \tau_{\mathrm{T}}=3 \times 10^{-3} . E_{i}$ corresponds to photon input at the third harmonic line. Most photons are redistributed by photon spawning to the wings of the fundamental line.

\subsection{X-ray pulsar continua}

In the following, we show cyclotron resonance scattering features in folded full spectra. As continuum input, we chose an exponentially cutoff power law of the form

$F(E)=A \cdot E^{-\alpha} \exp \left(-E / E_{\text {fold }}\right)$

where $A$ is the power law normalization, $\alpha$ the photon index and $E_{\text {fold }}$ the folding energy. This spectral shape is the most simple phenomenological model which qualitatively describes $\mathrm{X}$-ray pulsar spectra and is used here for illustrative purposes only. When considering real observational data, however, more complex continuum models like, e.g. a power law with a FermiDirac cutoff (Tanaka 1986) or a negative and positive powerlaw with exponential cutoff ("NPEX") (Mihara 1995) can be used. Only recently, an analytical derivation of the spectral shapes of X-ray pulsar spectra was presented by Becker \& Wolff (2005, 2007).

\section{Results}

In this section we make theoretical predictions from our Monte Carlo simulations and discuss their implications on observed properties of cyclotron lines. Special emphasis is placed on the study of the line parameters, i.e., line position, line width and line depth, of the fundamental CRSF.

\subsection{Geometry and optical depth}

To close the link to the publications by Araya \& Harding (1999) and Araya-Góchez \& Harding (2000), we illustrate in Figs. 4-6 full spectra folded with the convolution model for a chosen physical setting in a similar fashion as shown in their work: spectra are depicted for slab and cylinder geometries, for two different

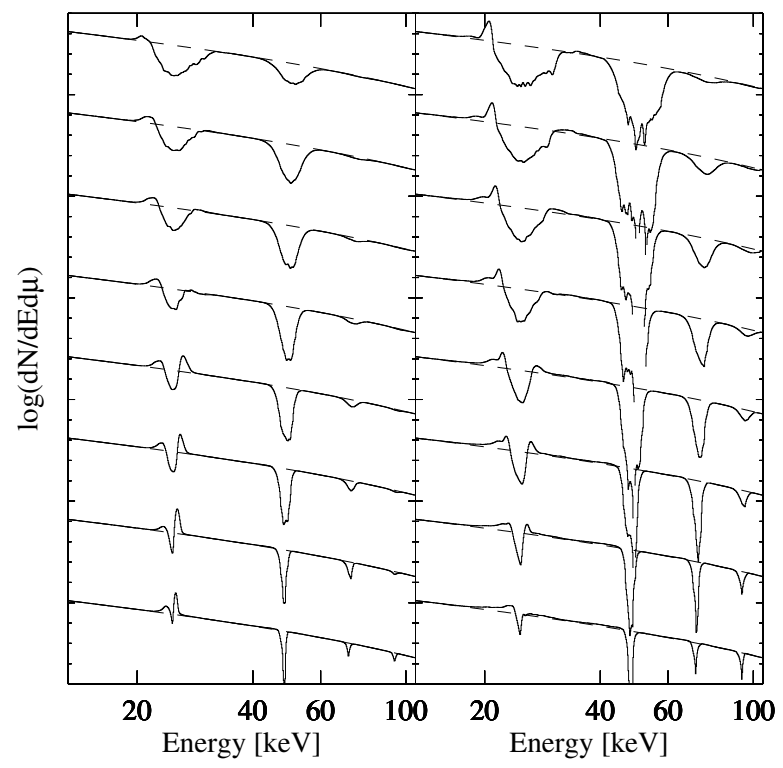

Fig. 4. Line profiles for cylinder geometry as a function of angle and optical depth. Left: continuum optical depth $\tau_{\mathrm{T}}=3 \times 10^{-4}$; right: $\tau_{\mathrm{T}}=3 \times 10^{-3}$. The emergent spectra [photons $\mathrm{ster}^{-1} \mathrm{~s}^{-1} \mathrm{keV}^{-1}$ ] from all eight angular bins are shown (bottom to top: $\mu=$ $\cos \theta \in[0.000,0.125),[0.125,0.250),[0.250,0.375),[0.375,0.500)$, $[0.500,0.625),[0.625,0.750),[0.750,0.875),[0.875,0.1000))$. In both panels, $B / B_{\text {crit }}=0.05$ and $k T_{\mathrm{e}}=3.0 \mathrm{keV}$. The continuum photon flux is assumed to have a power law distribution with photon index $\alpha=2.0$ and an exponential high energy cutoff at the folding energy $E_{\mathrm{fold}}=40 \mathrm{keV}$.

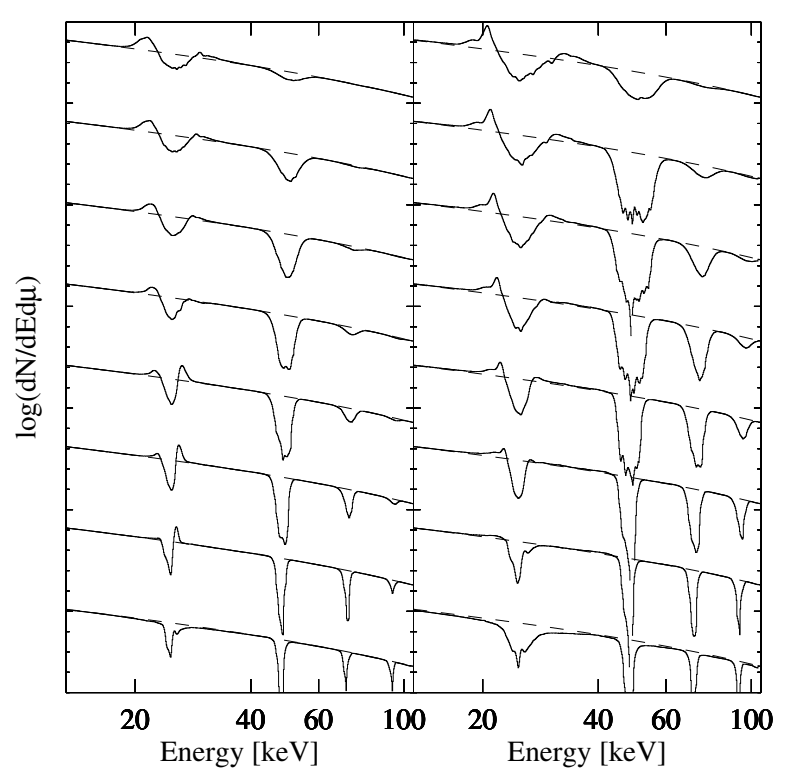

Fig. 5. Same as Fig. 4 for slab 1-1 geometry.

values of $\tau_{\mathrm{T}}$ and for different angular bins. The continuum parameters are fixed to $E_{\text {fold }}=40 \mathrm{keV}$ and $\alpha=2$. The choice of the folding energy describes a rather hard continuum, which leads to a pronounced contribution from high-energy photon spawning to the CRSFs (compare Sect. 4.5). Figures 4 and 5 are in agreement with the previous simulations of Araya \& Harding (1999). As an improvement with respect to the earlier results, we calculated the line shapes on a grid of 640 energy bins instead of 80 and we resolve the depicted line shapes for eight angular bins of $\cos \theta$ instead of four. The improved statistics of effectively $1.6 \times$ $10^{6}$ Monte Carlo photons per folded spectrum (increased from 


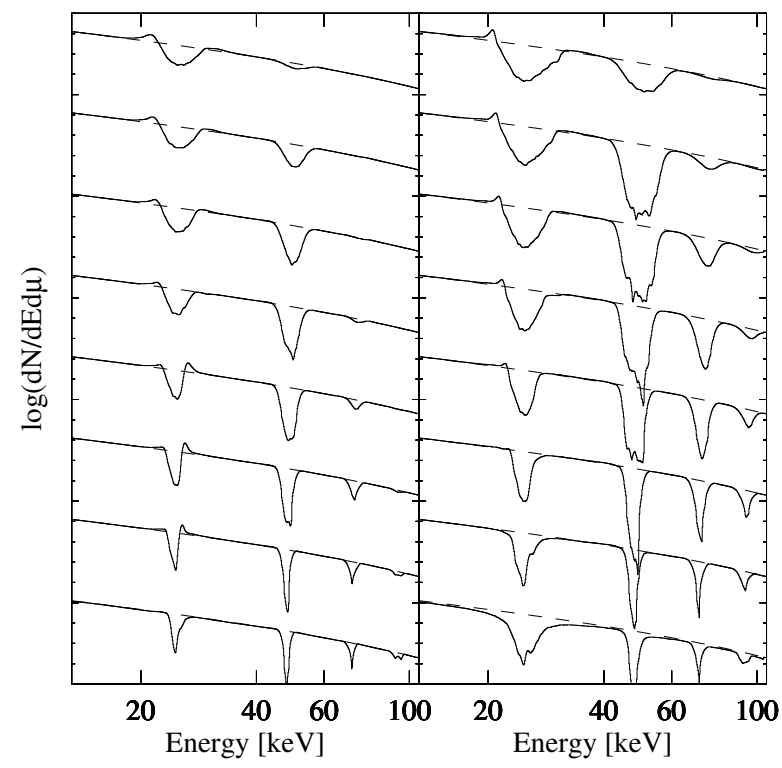

Fig. 6. Same as Fig. 4 for slab 1-0 geometry.

$5 \times 10^{4}$ ) gives us a well resolved picture of the line shapes, however, the centroid of the second harmonic would require even better statistics for continuum optical depths of $\tau_{\mathrm{T}} \sim 3 \times 10^{-3}$. Although the second harmonic is not fully described in its core, the calculations clearly show that the second harmonic is more pronounced than the fundamental one, which has a more complex, broad and shallow shape, often with emission wings, which are strongest for the internally illuminated plasmas. The strength of those emission wings is expected as a consequence of the source photon location at the mid-plane of a slab and mid-axis of a cylinder. For slab geometry, Isenberg et al. (1998b) and Nishimura (2005) showed comparisons of the slab 1-1 and the slab 1-0 geometry, and pointed out that these wings can be understood as the remnants of a strong emission feature forming near the mid-plane. For very small optical depths, $\tau_{\mathrm{T}}=10^{-4}$, we also find this feature in our simulations. Observations of sources with CRSFs have not been seen to exhibit strong emission wings. In Sect. 5.2, we will comment further on the observability of those features with modern instrumentation.

\subsection{Angular distribution}

Photons are injected isotropically into 20 angular bins in $\cos \theta$. Although the distribution of the initial photon directions is isotropic, a high degree of anisotropy arises after the photons have been propagated through the plasma due to a highly anisotropic scattering cross section. Figure 7 shows the angular redistribution of the photons for all considered geometries and for different values of the optical depth. Internally the code keeps track of twenty $\cos \theta$ bins. We show the angular redistribution resolved into eight final bins. For $\tau_{\mathrm{T}}=3 \times 10^{-4}$ we observe a trend of an overall redistribution towards smaller $\theta$ for slab geometry and a reverse trend for cylinder geometry. For a larger optical depth, $\tau_{\mathrm{T}}=3 \times 10^{-3}$, these trends increase for slab and decrease for cylinder geometry, where the curve in Fig. 7 flattens. This can be understood from the dependence of the scattering cross sections on the angle (see Fig. 2) which implies that there is general trend of a photon redistribution by scattering towards smaller angles, i.e., larger $\cos \theta$, regardless of the geometry. The larger the optical depth a photon must pass, the more scatterings take place and the more dominant this effect becomes. This condition can

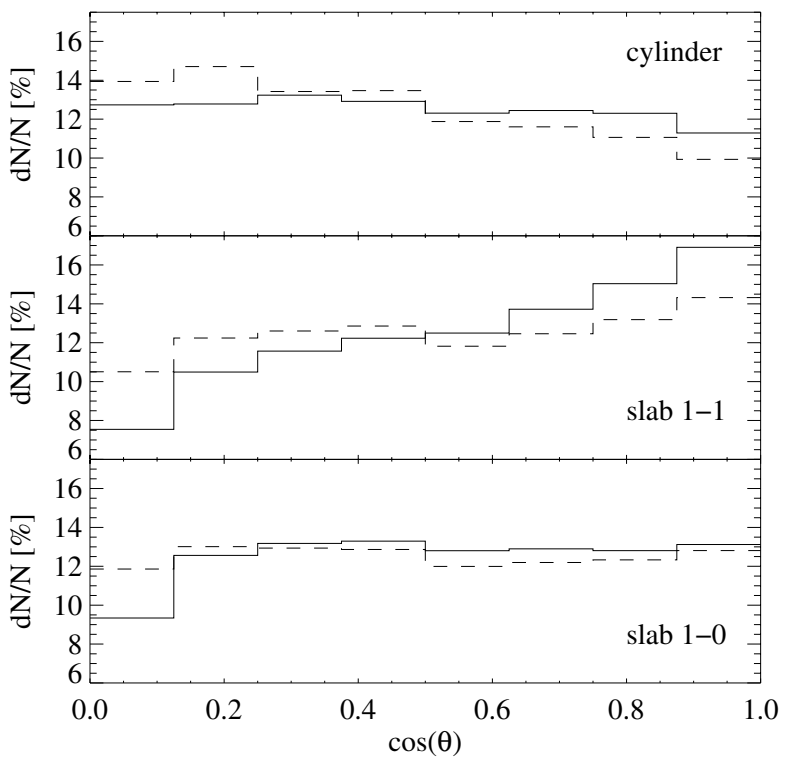

Fig. 7. Angular redistribution of the photons. For isotropic photon injection, the percentage of the emitted photon flux into eight angular bins for the spectra in Figs. 4-6 is shown. Top: cylinder geometry, middle: slab 1-1 geometry, bottom: slab 1-0 geometry. Solid lines: $\tau_{\mathrm{T}}=3 \times$ $10^{-3}$, dashed lines: $\tau_{\mathrm{T}}=3 \times 10^{-4}$.

also account for a less prominent trend in the slab 1-0 geometry compared to the 1-1 geometry, as photons which experience many scatters by various crossings of the source plane are thermalized, biasing the emerging radiation. For a fixed optical depth $\tau_{\mathrm{T}}=1 \times 10^{-3}$ similar plots are shown resolved into all twenty bins by Araya-Góchez \& Harding (2000). For $\tau_{\mathrm{T}} \sim 8 \times 10^{-4}$, the angular photon redistribution of the line photons is discussed by Isenberg et al. (1998b). Due to the redistribution of photons in angle, the cyclotron line shapes also vary significantly. We have fitted the fundamental feature with the continuum spectral function multiplied with a Lorentzian in absorption for the absorption feature and two Lorentzians in emission for the emission wings. Figure 8 shows characteristic parameters of the fundamental CRSF as obtained from these phenomenological fits.

The line position of the fundamental CRSF varies little with the viewing angle and thus cannot account for the amount of change in line positions observed for some sources with phase or during various observations. Therefore, different explanations have to be sought. A possible scenario could be a variation of the magnetic field with angle. The line depth increases with $\cos \theta$ in the case of cylinder geometry, and decreases for slab geometry. This is easily understood from Eqs. (9) and (10), as they predict the largest optical depth for small $\theta$ for cylinder, and for large $\theta$ for slab geometry. For cylinder geometry, the line width increases clearly with $\cos \theta$ as expected from the angledependence of relativistic Doppler broadening (see Eq. (13) below) and reinforced by the increasing optical depth with $\cos \theta$. The decreasing optical depth with angle for slab geometry instead suppresses the trend of the line broadening with $\cos \theta$.

\subsection{Line energies vs. magnetic field strength $B$}

Line profiles for different magnetic field strengths and otherwise fixed parameters are shown in Fig. 9. The figure clearly shows the approximately linear progression of the centroid line energies towards higher energies with increasing magnetic field strength expected from the $12-B-12$ rule. The relativistically 


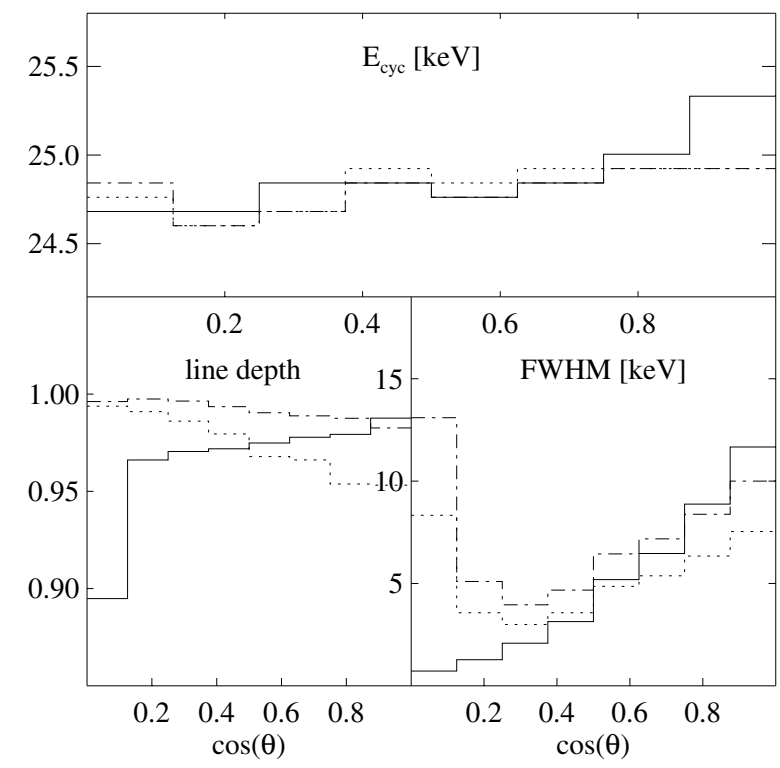

Fig. 8. Variation of the line parameters of the fundamental CRSF with the angle of emission. Solid lines: cylinder geometry, dashed lines: slab 1-1 geometry, dash-dotted lines: slab 1-0 geometry. All values are obtained from fits of the line shapes depicted in Figs. 4-6, and for a Thompson optical depth of $\tau_{\mathrm{T}}=3 \times 10^{-3}$.

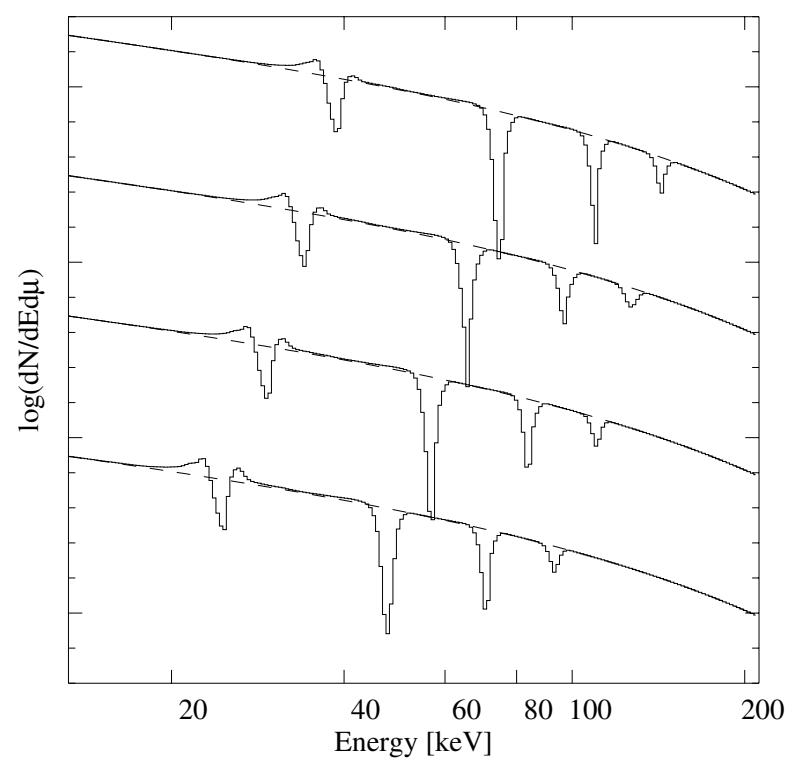

Fig. 9. Line profiles for different values of the magnetic field strength. The $B$-field increases from bottom to top: $B / B_{\text {crit }}=$ $0.05,0.06,0.07,0.08$. We assume cylinder geometry, a constant temperature $k T_{\mathrm{e}}=3 \mathrm{keV}$, an optical depth $\tau_{\mathrm{T}}=3 \times 10^{-3}$ and a viewing angle $\theta$ with $\mu=\cos \theta=0.25$. The continuum spectrum has the shape of a power law with photon index $\alpha=2.0$ and with an exponential cutoff at the energy $E_{\mathrm{fold}}=40 \mathrm{keV}$.

correct line ratios for a uniform field are implied by Eq. (3). For some sources, e.g., V0332+53 (Pottschmidt et al. 2005) this behavior is confirmed by observational data. As mentioned above, there are also sources for which the line energies of the harmonics are much less harmonically spaced than can be accounted for by relativistic effects. One possible explanation is a model in which the magnetic field is varying locally within the lineforming region. Estimates of the dipole magnetic field of pulsars from torque theory and measurements of the surface magnetic

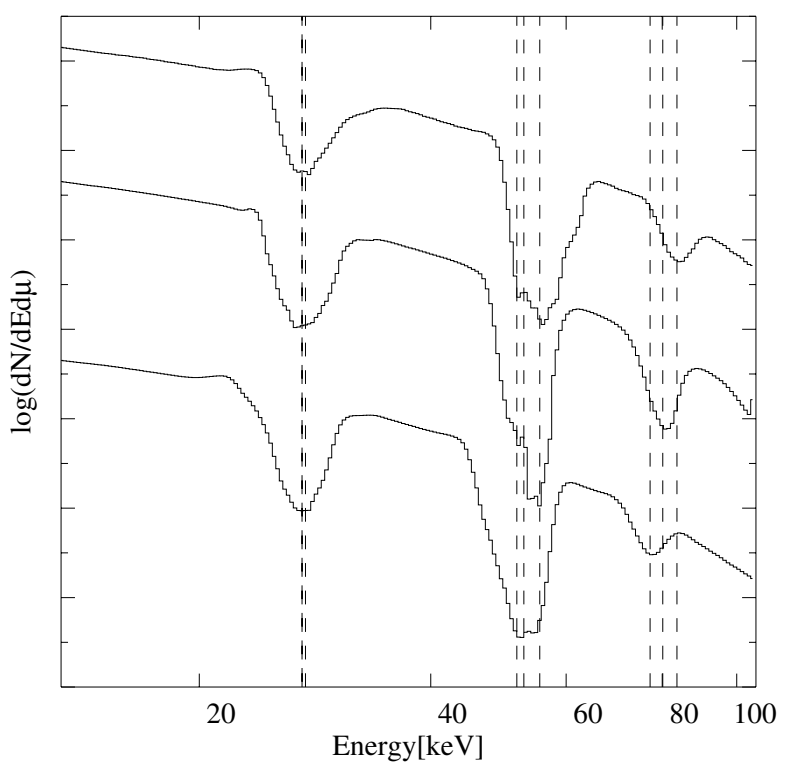

Fig. 10. Comparison of CRSFs for a uniform and a non-uniform magnetic field in a line-forming region of slab 1-0 geometry. Bottom: $B / B_{\text {crit }}=0.05 \rightarrow 0.055$, middle: $B / B_{\text {crit }}=$ const. $=0.055$, top: $B / B_{\text {crit }}=0.06 \rightarrow 0.055$. Results are shown for $\mu=\cos \theta=0.6$. Otherwise the same setting as in Fig. 9 is used. The vertical dashed lines mark the line positions obtained from a phenomenological fit (continuum multiplied by three Lorentzians in absorption for the lines and two Lorentzians in emission for the emission wings of the fundamental line) of the first three CRSFs.

field from CRSF detections hint at a magnetic field of more complex structure than a simple dipole. As the exact nature of the magnetic field is unknown, different scenarios for non-dipole magnetic field structure have been investigated (Blandford et al. 1983; Urpin et al. 1986; Arons 1993). Besides magnetic field gradients which arise due to dipole variations with the height of the magnetosphere, surface field variations can result, e.g., from small-scale crustal field structures (Blandford et al. 1983), or thermomagnetic field evolution effects (Urpin et al. 1986). Nishimura (2005) recently presented a study of the line ratios for a line-forming region of slab geometry threaded by a magnetic field which linearly varies with the height. Their approach was based on a model by Gil et al. (2002) who assumed the presence of a star-centered dipole from a fossil field in the core superposed by a crust-anchored dipole anomaly from crustal field structures. Applying Feautrier methods to solve the radiative transfer, Nishimura (2005) found that the line ratios significantly increase if the $B$-field decreases upwards, and decrease vice versa. We have adapted this toy model for a first study of magnetic field spread with our Monte Carlo approach. With some simplifying assumptions considering the geometry and the angular redistribution for the case of a non-constant $B$-field, we can confirm the trend of line-ratio increase and decrease proposed by Nishimura (2005). An example is shown in Fig. 10, where we compare cyclotron lines for a constant, a linearly decreasing, and a linearly increasing magnetic field. For the non-constant magnetic field we prescribe a linear variation of the field strength in discrete steps within the line forming region of up to $10 \%$. The fundamental line appears widely unchanged in shape and position, as it is formed in the upper scattering layers, where the nonconstant $B$-field was set to have the same value as the constant one. Line photons which have been scattered out of the line of sight or redistributed in energy in lower layers are replaced by spawned photons from scattering in higher layers. By contrast, 
Table 1. Comparision of line positions and line ratios from the fits of the spectra shown in Fig. 10. The line energies are given in keV. For the case of a constant magnetic field, the line energies are also calculated from Eq. (3).

\begin{tabular}{ccccc}
\hline \hline$B / B_{\text {crit }}$ & 1st & 2nd & 3rd & Line ratios \\
\hline $0.050 \rightarrow 0.055$ & 27.20 & 51.76 & 77.16 & $2.84: 1.90: 1$ \\
0.055 & 27.20 & 52.87 & 80.14 & $2.95: 1.94: 1$ \\
$0.060 \rightarrow 0.055$ & 27.49 & 55.45 & 83.61 & $3.04: 2.02: 1$ \\
0.055 (Eq. (3)) & 27.62 & 54.35 & 80.27 & $2.91: 1.96: 1$ \\
\hline
\end{tabular}

the higher harmonic lines change in position and shape. Here, contributions from all layers of different depth are important for the final line profile. Absorption features from photons at low layers are not refilled. Hence, with the change of the resonant energies with the height of the line forming region the lines become wider (proportional to the amount of variation in $B$ ) and their final centroid energy is shifted. From fitting Lorentzians to the first three lines we obtained the line energies and line ratios for a constant, increasing and decreasing magnetic field. In Table 1 we list the fitted line positions and the fitted line ratios. The theoretical values of the line energies and line ratios after Eq. (3) are shown for completeness. As expected, these values are similar to the fitted ones for a constant magnetic field, although not identical, as the non-Gaussian and non-Lorentzian line shapes especially of the fundamental line restrict the fit quality noticeably.

Line ratios of strongly anharmonic nature have been observed. For $4 U$ $0115+63$, the observed spacing of the line energies is smaller than expected, yielding line ratios of $(2.8 \pm$ $0.05: 1.9 \pm 0.05: 1)$ (Santangelo et al. 1999) or (2.71 \pm 0.13:1.73 \pm 0.08:1) (Heindl et al. 1999) for the first three harmonics. These deviations are comparable to the ones we obtained for an increasing magnetic field in our example. Using a different modeling approach, and tuning his parameters to the case of 4 U $0115+63$, Nishimura (2005) has already shown that these line ratios can be reproduced by the assumption of a variable magnetic field in the line forming region. An example for a source where the line ratios may be higher than what is expected from Eq. (3) is Vela X-1, where Kreykenbohm et al. (1999, 2002) have found a coupling of the first harmonic energy to the fundamental line energy $\gtrsim 2$ in $R X T E$ data.

\subsection{Influence of the plasma temperature}

The line width is determined by the energy and angle-dependent shape of the scattering cross section and smeared out due to thermal Doppler broadening. In Fig. 11, cyclotron line shapes are depicted for varying parallel electron temperature $T_{\mathrm{e}}$, for cylinder geometry, fixed parameters $B, \cos \theta$ and for a fixed continuum shape. The hotter the plasma, the wider and the more asymmetric become the lines. The width of the lines is due to a combination of the natural line width and Doppler broadening (Harding \& Lai 2006). Doppler broadening gives a Full Width Half Maximum of (Trümper et al. 1977; Mészáros \& Nagel 1985)

$\Gamma_{\text {FWHM }}=\sqrt{\frac{8 \ln (2) k T_{\mathrm{e}}}{m_{\mathrm{e}} c^{2}}}|\cos \theta| E_{\mathrm{cyc}}$.

For increasing $\cos \theta$ the line shapes become more asymmetric. In our simulations the plasma temperature is a free parameter. From theoretical (Lamb et al. 1990; Isenberg et al. 1998b) and

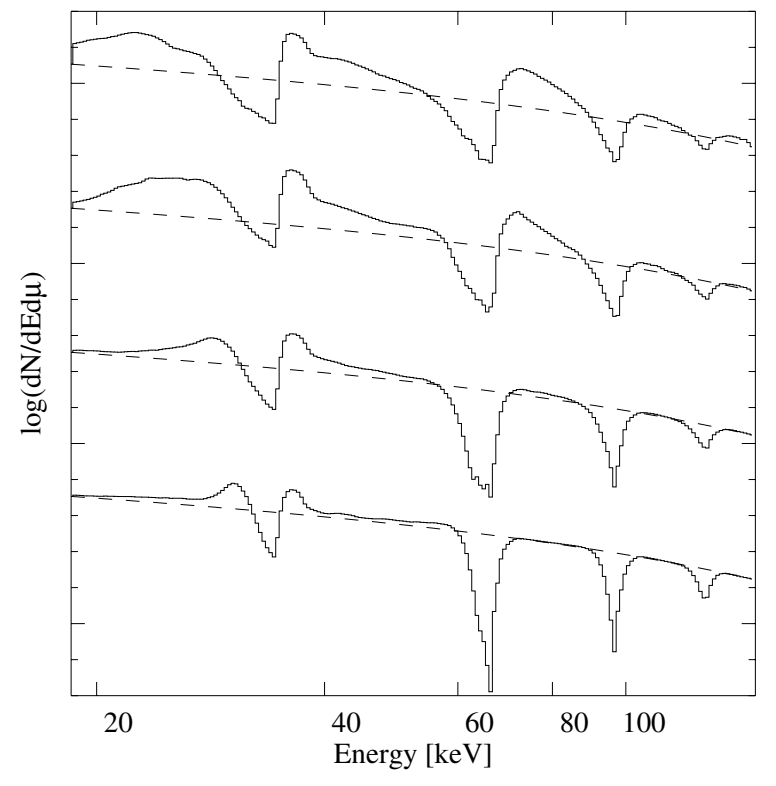

Fig. 11. Variation of the line shapes with the temperature $\left(k T_{\mathrm{e}}=\right.$ $5,10,15,20 \mathrm{keV}$ from bottom to top). Spectra are shown for a magnetic field $B / B_{\text {crit }}=0.07$ and otherwise the same parameters as in Fig. 9 .

observational studies (Heindl et al. 2004), a relation between observed magnetic field and temperature has been proposed as

$k T_{\mathrm{e}} \gtrsim 0.2 E_{\text {cyc }}$.

This would correspond to a temperature for the depicted setting $\left(E_{\mathrm{cyc}} \sim 30 \mathrm{keV}\right)$ of at least $6 \mathrm{keV}$, i.e. somewhere in between the bottom spectrum and the second spectrum from the bottom in Fig. 11.

\subsection{Continuum shape and photon spawning}

The shape of the cyclotron lines is sensitive to the continuum shape. In particular, the fundamental line shape and its emission features vary significantly. For better illustration we show the case of internally irradiated plasmas where the emission wings are strongest. The dependence of the line shapes on the continuum can be understood when considering the photon redistribution in energy, especially due to photon spawning. Figure 12 shows the change of the line profiles for a flat input continuum spectrum, when allowing only for electron transitions between the ground Landau state to the first Landau level, or for photonelectron scattering leading to up to three harmonics. In the former case, a single absorption line forms. The more harmonic scatterings are allowed for, the more lines form, while the fundamental and lower harmonics become shallower with growing emission wings. Integrating the photon flux only over the energy range including just the fundamental line and its emission wings ( $E \leq 18 \cdot \mathrm{keV} B_{12}$ ), we find that the spawned photons account for as much as $34 / 64 / 73 \%(n \leq 2,3,4)$ of the flux for cylinder and for $11 / 32 / 43 \%$ of the flux for slab $1-1$ geometry. Considering the whole energy range, the percentage of spawned photons is $25 / 52 / 65 \%$ of the total flux for cylinder and $3 / 15 / 25 \%$ of the total flux for slab geometry. Note that these numbers are representative of the extreme and fairly unrealistic case of a flat input continuum. However, they illustrate well that the line shapes should change with the spectral hardness of the incident continuum, where harder spectra exhibit more emission features near shallower lines. Line profiles for a power law with a photon in$\operatorname{dex} \alpha=1$ with exponential rolloff at different folding energies 


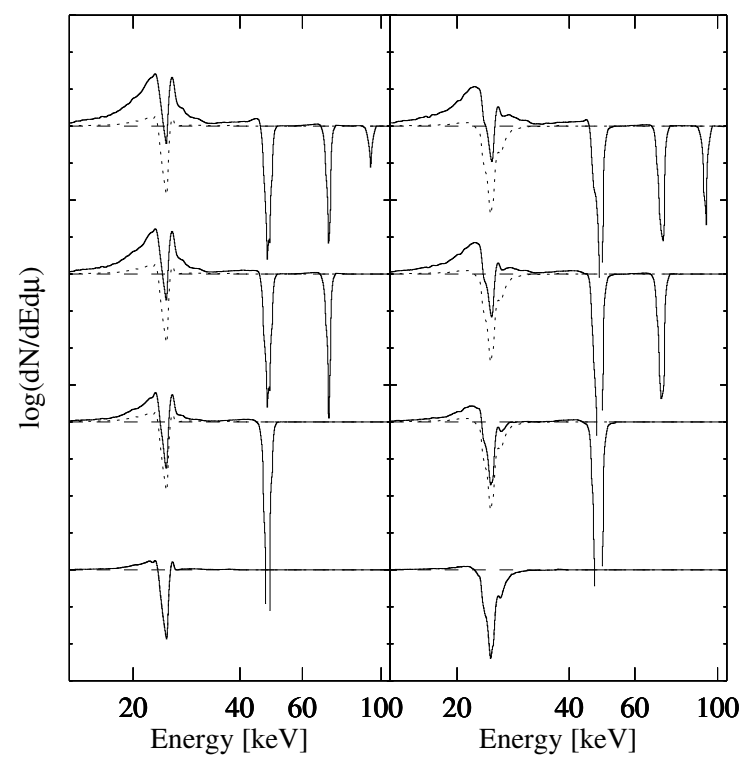

Fig. 12. Changes of the line profiles for consideration of one to three harmonics for the scattering processes (bottom to top). The bottom graph is overplotted in all cases as a dotted line to guide the eye. The input continuum spectrum (dashed line) is flat and therefore overemphasizes the effects of emission wings. Left panel: cylinder geometry; right panel: slab 1-1 geometry. In both panels, $k T_{\mathrm{e}}=3 \mathrm{keV}$, $\cos \theta \in[0.125,0.250), \tau_{\mathrm{T}}=3 \times 10^{-3}$ and $B / B_{\text {crit }}=0.05$.

$E_{\text {fold }}=5,15 \mathrm{keV}$, and for a pure power law continuum spectrum are shown in Fig. 13. In this case, the number of photons around the first line increases by a factor of 1.1 and 2.2 for the two harder spectra with respect to the softer one.

\section{Applications}

\subsection{A new XSPEC model for cyclotron lines: cyclomc}

At present, a physical model for the analysis of cyclotron lines is lacking. Typically, for each line independently, multiplicative phenomenological model components such as Gaussian, or Lorentzian absorption lines are used. They yield simplified, smoothed line shapes; various CRSFs are fitted independently from each other one by one. Although it is possible to fit observed data well with this phenomenological approach, the loss of physical information is very unsatisfactory. Overlaying several Gaussians or Lorentzians renders it also difficult to infer the magnetic field strength with the line centroid position, and does not permit one to distinguish, e.g., thermal and other broadening of the CRSFs. The fact that the line positions are independent of each other in this approach invokes further information loss. With the increasing quality of the energy resolution of todays' detectors it is possible to see more details of the line features calling for a physical model instead of a phenomenological one.

For purposes of data analysis, we have implemented a cyclotron line convolution model called cyclomc as a local model for fitting CRSFs in XSPEC. Based on the Green's functions of Sect. 3.4, cyclomc is designed to fit up to four CRSFs simultaneously. Not only the magnetic field strength and the temperature, but also the optical depth and the ratios of line positions, line widths and line depths are determined by the underlying physical picture. The quality of the fit hence permits conclusions on the accuracy of this picture. As shown in Sect. 4.3, the line ratios could be a sensible indicator for instance for magnetic field variations along the line of photon propagation. The model is

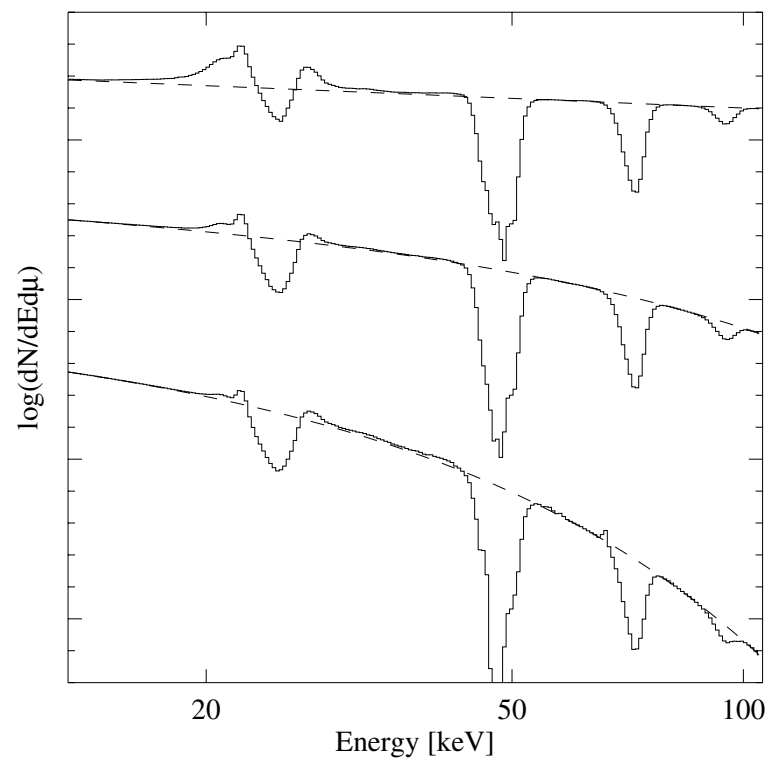

Fig. 13. Line profiles for different continua. All continua have the general form of a power law with a photon index $\alpha=1.0$ (top). In the middle and bottom plots, the spectra have an additional high energy cutoff at the folding energies $E_{\text {fold }}=15 \mathrm{keV}$ for the middle and $E_{\text {fold }}=5 \mathrm{keV}$ for the bottom plot. We show the results for cylinder geometry, $k T_{\mathrm{e}}=3 \mathrm{keV}, \cos \theta \in[0.375,0.500), \tau_{\mathrm{T}}=3 \times 10^{-3}$, and $B / B_{\text {crit }}=0.05$.

obviously restricted to the physical assumptions and the parameter input chosen for the Monte Carlo simulations. During fitting, it interpolates the results on the assumed input parameter grid which has been described in Sect. 3.4. Since cyclomc is a convolution model, self-consistent line shapes are obtained for any given continuum.

\subsection{Observability of the line features}

Before applying cyclomc to an example of observational data, we want to address the more general question of the observability of the predicted CRSFs in real source data. It is a fundamental question to assess which features of the model still appear when folded with the detector responses of todays' observatories. This question had already been posed by Isenberg et al. (1998b) considering the issue of different geometries and densities related to the prediction of significant emission wings. However, we are not aware of a related study up to date. In Fig. 14, we show the simulated spectrum for a source which we assign an ideal spectrum of the form of the npex model (Mihara 1995; Makishima et al. 1999), a negative and positive power law with a common high-energy cutoff,

$F(E)=A\left(E^{-\alpha_{1}}+f \cdot E^{+\alpha_{2}}\right) \exp \left(-\frac{E}{E_{\text {fold }}}\right)$,

folded with a chosen set of the Green's functions cyclomc for slab 1-1 geometry. The flux is appropriate for typical HMXB observations. For an assumed observation time of $20 \mathrm{ks}$ this spectrum was folded with the RXTE HEXTE response and background was added. We then fitted our fake spectrum, using a npex component for the continuum and two Gaussian absorption lines (Coburn et al. 2002) to model the simulated CRSFs. Figure 14 shows this fit as well as the residuals for a fit of the continuum component and for a fit of continuum and line components. The residuals from the continuum shape represent our 


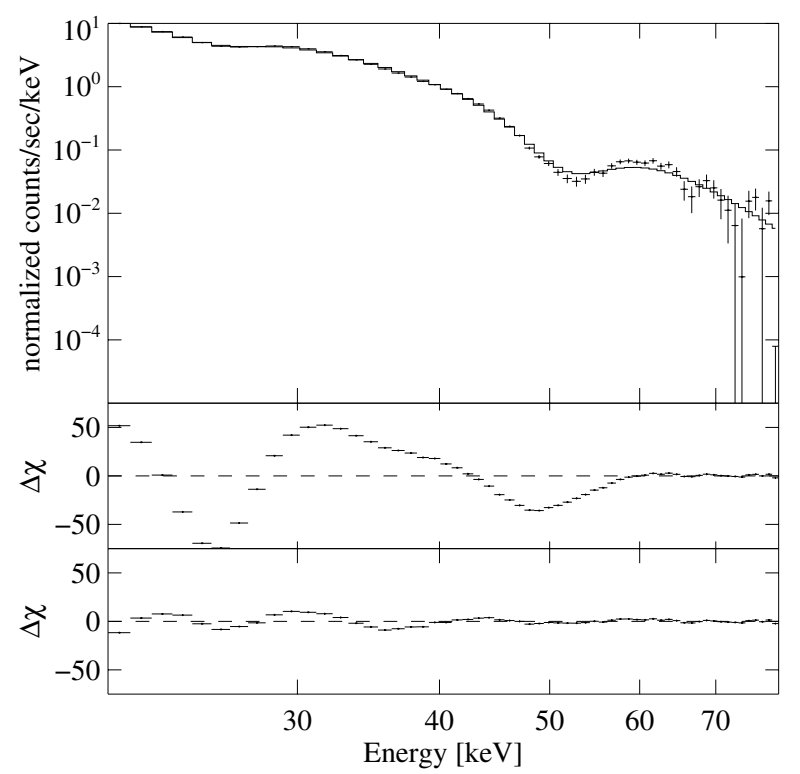

Fig. 14. HEXTE simulations. Upper panel: a simulated spectrum of the form npex $\cdot$ cyclomc (slab 1-1 geometry, $B_{12}=3.0, k T_{\mathrm{e}}=5.5 \mathrm{keV}$, $\tau_{\mathrm{T}}=5 \times 10^{-4}, \cos \theta=0.5, z=0.3, \alpha_{1}=1.5, \alpha_{2}=1.5, E_{\mathrm{fold}}=$ $\left.6.16 \mathrm{keV}, A=4.6 \times 10^{-3}, f=1.17\right)$ is fitted with a npex multiplied with two Gaussian absorption lines (XSPEC: gauabs). Upper panel: data and fitted model. Middle panel: residuals for the simulated spectrum fitted with a npex continuum. Bottom panel: residuals for the continuum and lines fit shown in the upper panel. The strong emission wings of the fundamental line in the simulated spectrum are clearly observable in the residuals.

simulated line shapes as we have used the same continuum component for the simulated and for the model spectrum. Fitting the first two lines with Gaussians, the emission wings stay very pronounced in the residuals. For our simulated spectrum we expect to observe the fundamental CRSF at $E_{1}^{\text {obs }}=26.1 \mathrm{keV}\left(B_{12}=3.0\right.$, $z=0.3, \cos \theta=0.5)$. The Gaussian fit of the fundamental line gives a centroid energy of $24.77 \pm 0.03 \mathrm{keV}$ instead. This results indicates that the asymmetric line shapes could introduce a systematic uncertainty in line energy when modeling observed data with Gaussian or Lorentzian shapes. We also note that the lines seem very prominent even for low values of continuum optical depth.

\subsection{Comparison to observational data}

During the recent outbursts of the transient sources V0332+53 and A0535+26 (Pottschmidt et al. 2005; Kreykenbohm et al. 2005; Kretschmar et al. 2005; Terada et al. 2006) more data from accreting X-ray pulsar systems containing strong cyclotron resonance scattering features were collected. We have fitted $R X T E$ and INTEGRAL data of the January 2005 outburst of V0332+53 as a first example for the comparison of cyclomc with real source data. Discovered by Tanaka (1983), V0332+53 was the fourth accreting X-ray pulsar system where CRSFs were found (Makishima et al. 1990a,b). Its third CRSF was discovered in RXTE data (Coburn et al. 2005; Pottschmidt et al. 2005) and confirmed by subsequent INTEGRAL observations (Kreykenbohm et al. 2005). In Fig. 15 we show a fit of the first two CRSFs in the INTEGRAL data of $\mathrm{V} 0332+53$. The fit of the RXTE data of $\mathrm{V} 0332+53$ during the same outburst is not shown as the principal

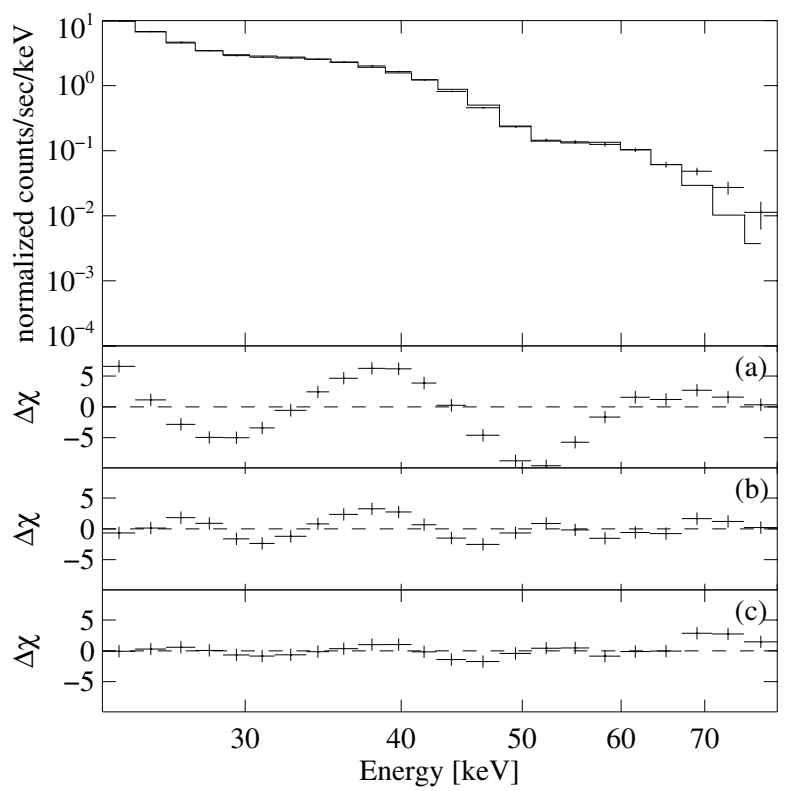

Fig. 15. Fit of the Green's functions CRSF model cyclomc to the line features of a phase-averaged spectrum of V0332+53 obtained from INTEGRAL IBIS observations. The data and fitted model (upper panel) along with $\Delta \chi$ residuals are shown (three lower panels). The residuals are depicted for a) a continuum fit, and for two different continuum and line fits using b) slab 1-1 geometry, and c) slab 1-0 geometry combined with partial covering. The continuum shape is taken as a power law with a Fermi-Dirac cutoff (fdcut). For the best fit parameters, see Table 2.

results turned out very similar for both instruments. We modeled the data with a power law with a Fermi-Dirac cutoff (fdcut),

$F(E)=A \cdot E^{-\alpha}\left[\exp \left(\frac{E_{\text {cut }}-E}{E_{\text {fold }}}\right)+1\right]^{-1}$,

folded with the CRSF Green's functions (cyclomc) for different geometries, and assuming 5\% systematics for the IBIS data. The best fit was obtained for the slab 1-0 geometry in combination with the scenario of partial covering and gave a reduced $\chi_{\text {red }}^{2}$ of 2.2 for 13 degrees of freedom. Figure 15 shows the best-fit model along with the data and the residuals for fitting the continuum component, fitting cyclomc for slab 1-1 geometry, and fitting cyclomc for slab 1-0 geometry, combined with partial covering, to the data. The best-fit parameters are listed in Table 2. While the slab 1-0 geometry is set apart from the other geometries by exhibiting the weakest emission wings, the scenario of partial covering effectively reduces the line depths of the model spectrum (compare Sect. 5.2). We stress that these fits are preliminary, serving at this stage of the model design as a proof of concept only and as an important first step to help us in understanding the physics of CRSF formation. The rather high $\chi^{2}$ should be seen in the light that this is the first time that a realistic CRSF model has been fit to real observational data. The fact that we can assess the physics via a direct comparison to data enables us to draw conclusions concerning the underlying physical picture. First of all, the use of the 1-1 geometry results in emission wings which are clearly too pronounced for the observed data. We have shown in Sect. 5.2 that a simulated source with a spectrum identical to the theoretical one would indeed still exhibit detectable emission wings in real data. These strong emission features are clearly not present in the spectrum of V0332+53. We therefore conclude that the 1-1 geometry of an internally irradiated plasma is not a completely valid physical assumption. 
Table 2. Best fit parameters for the data and model shown in Fig. 15. Uncertainties are at the $90 \%$ confidence level for one interesting parameter; $\chi^{2}=28.4$ for 13 d.o.f.

\begin{tabular}{ll}
\hline \hline Name [unit] & Fit value \\
\hline$B_{12}\left[B /\left(10^{12} \mathrm{G}\right)\right]$ & $3.05_{-0.03}^{+0.05}$ \\
$k T_{\mathrm{e}}[\mathrm{keV}]$ & $10.2_{-0.3}^{+0.3}$ \\
$\tau_{\mathrm{T}}\left[10^{-3}\right]$ & $3.0_{-0.4}^{+0}$ \\
$\cos \theta$ & $0.06_{-0}^{+0.02}$ \\
$z$ & $0.23_{-0.02}^{+0.02}$ \\
$\alpha$ & $0.94_{-0.04}^{+0.08}$ \\
$E_{\text {cut }}[\mathrm{keV}]$ & $12.8_{-3.3}^{+1.4}$ \\
$E_{\text {fold }}[\mathrm{keV}]$ & $7.5_{-0.1}^{+0.1}$ \\
$A_{1}$ & $2.1_{-3.8}^{+8.7}$ \\
$A_{2}$ & $0.87_{-0.05}^{+0.04}$ \\
\hline
\end{tabular}

Considering the source photons to be injected at the bottom of the slab (1-0 geometry) or according to a probably biased distribution of incident photon production in the plasma seems to be more realistic. Second, we see as a general problem with the fit that the line depths cyclomc yields for rather low $\tau_{\mathrm{T}}$ are very deep compared to the observations. The scenario of partial covering of the emergent radiation can solve this issue. In this case, only part of the emergent radiation is assumed to pass the region of line formation and is reprocessed with cyclomc. A different possibility to ensure shallower line depths, however, would be the introduction of a magnetic field gradient along the $B$-field vector in a cylindrical line-forming region (O. Nishimura, private communication). Besides yielding shallower lines, such a scenario might also improve the fit due to variations in the line ratios.

\section{Summary and discussion}

We have performed an in-depth study of the formation of cyclotron resonance scattering features in the spectra of highly magnetized accreting neutron stars. In particular, we have discussed the influence of the magnetic field strength, the plasma temperature, the angle of radiation and the seed continuum spectral form onto the shapes of cyclotron line features.

Our study, being based on the Monte Carlo simulation code of Araya \& Harding $(1996,1999)$ and Araya-Góchez \& Harding (2000), emphasizes several issues which have been pointed out by those authors before. Examples are Figs. 4 and 5, where we have discussed the optical depth progression matching our illustrations to Figs. 4-6 from Araya \& Harding (1999) or Fig. 7 for the study of angular redistribution similar to the illustrations used in Araya-Góchez \& Harding (2000). With respect to their previous code our model is different in the following points: most importantly, we did not restrict ourselves to the study of hard continua, but chose a Green's functions approach, thus gaining independence from a priori chosen forms of incident radiation. We relaxed the geometrical constraints on the line-forming region to include the case of a bottom-illuminated slab as it has been studied by, e.g., Isenberg et al. (1998b) and Nishimura (2005). Moreover, the higher resolution in angle and energy binning permits to illuminate the complex form of the fundamental feature in detail. The time-consuming calculations on a huge parameter grid in principal allow for a systematic comparison of our results to observational data, which is facilitated by the implementation of our local model cyclomc for XSPEC.
Beyond this, we were able to confirm several results from other authors obtained with different numerical approaches. In Fig. 7 (Sect. 2.1) we have shown the angular redistribution of photons for cylinder and slab geometry and for two different values of the plasma optical depth. The percentage of redistributed photons per $(\cos \theta)$-bin increases with $\theta$ for cylinder and decreases with $\theta$ for slab geometry as shown in a similar study of an internally irradiated line forming region by Araya-Góchez \& Harding (2000). For a higher optical depth and for cylinder geometry, however, the photon distribution flattens, similar to results shown by Isenberg et al. (1998b) for the comparable case of $\Phi=\pi / 2$ and slab 1-1 geometry. The study of the variation of the cyclotron line ratios for a non-uniform magnetic field picks up an idea from Nishimura (2005), who investigated this case for a $B$-field which varies linearly with height, slab geometry, and for similar optical depths. We have confirmed the trend of an increase of the line ratios with a decreasing magnetic field and a decrease of the line ratios with an increasing magnetic field within a line-forming region of slab 1-0 geometry (Fig. 10).

In Sect. 2.1 we reported theoretical predictions of the model independently from observational data analysis. Except for the study of the line ratios, outlined above, all analysis assumed a uniform magnetic field in the line-forming region. A key result is the study of the variation of the line parameters of the fundamental feature with the magnetic field (Fig. 9), optical depth and angle (Figs. 4-8), and temperature (Fig. 11), which is consistent with predictions from other authors. We do not see a significant variation of the fundamental line energy, $E_{\text {cyc }}$, with the angle, and thus must exclude this simple scenario as an explanation for observed phase dependent variations of $E_{\text {cyc. }}$. Omitting the emission wings, the depth of the fundamental with respect to the continuum flux is rather stable over $\theta$ whereas the line width varies significantly with $\theta$ for cylinder and slab geometry. For both geometries the lines become wider towards higher $\cos \theta$; for slab geometry an initial decrease for $\cos \theta<0.25$ is observed. The variation of the overall line features for different magnetic field strengths and different temperatures was investigated. Obviously, the positions of the CRSFs are directly linked to the $B$-field strength (see Eqs. (1) and (3)). However, Fig. 9 also shows that changes with $B$ as to the line shapes are rather insignificant. On the other hand, the line shapes vary strongly with increasing temperature, where more asymmetric, Doppler-broadened lines arise for higher plasma temperatures. Furthermore, we have studied in depth variations of the line shapes with the incident continuum shape. In particular, the shape of the fundamental line changes with the continuum shape, an effect which can be understood from photon redistribution, mainly due to photon spawning in hard continua. As a result, for hard spectra for instance the emission wings are much more pronounced than for softer continua. This dependence of the CRSFs on the continuum in principle also allows for conclusions on the continuum shape when modeling cyclotron resonance scattering features.

This study aims at meeting the interests of observers in analyzing cyclotron lines. We therefore chose a broad approach to the topic of line formation. Fitting CRSFs in V0332+53 with cyclomc indicates that we can indeed assess CRSFs in real observed source data with a physical model. This is the first time such a simultaneous fit of several CRSFs with a realistic, physical model has been attempted. As outlined in Sect. 5.3, modifications of the underlying physical scenario guided by these preliminary fits should help in further improving the comparison of the model and real data. In Sect. 5.2 we have assessed the general question of the observability of the theoretically 
predicted line shapes. At the early stages of cyclotron line observations it was not clear whether emission features - if they were present in the data - would be observed or just smeared out by the detectors. For instance Isenberg et al. (1998b) and Nishimura (2005) observed that the scenario of a radiation source at the bottom of a slab as geometry for the line forming region leads to less emission features in the spectra than an internally irradiated plasma. We have shown in Sect. 5.2 that such strong emission features as predicted by our scenario should indeed be observable by the instruments on todays' observatories. The fit results from $\mathrm{V} 0332+53$ data demonstrate that large emission wings can be ruled out for this spectrum.

This work is ongoing, aiming at further generalizations of the CRSF model. We intend to permit angular anisotropy of the continuum photon flux. Araya-Góchez \& Harding (2000) have already studied this case for special angular distributions like peaked emission. We strive for the calculation of our Green's functions independently not only of the continuum energy but also of the continuum angular distribution to generalize our model to arbitrary angular distributions of the incident photons. The first issue to be investigated further, however, will be the observed discrepancy in theoretical and observational line depths. More realistic $B$-field gradients could account for shallower lines. We have shown that the consideration of a scenario of partial covering, which effectively reduces the line depths, significantly improves the fit quality. We therefore think that the consideration of non-constant magnetic fields within the line forming region will play a major role in the process of better modeling and understanding cyclotron lines. This issue will be subject of a forthcoming paper once having realized a systematic comparison of cyclomc for different scenarios for line formation to a larger set of observational data.

Acknowledgements. We thank Rafael Araya for stimulating discussions and for providing the first version of the Monte Carlo code used here. We also thank the Department of Physics of the University of Warwick and the European Space Astronomy Centre of the European Space Agency for their hospitality. We acknowledge the Centre for Scientific Computing of the University of Warwick and the Regionales Rechenzentrum Erlangen for providing the computing resources used in this work. This work was supported by a scholarship from the Studienstiftung des Deutschen Volkes and by the DLR grant 50 OR 0302. The observability analysis was based on RXTE data. The data comparison was based on observations with INTEGRAL, an ESA project with instruments and science data centre funded by ESA member states (especially the PI countries: Denmark, France, Germany, Italy, Switzerland, Spain), Czech Republic and Poland, and with the participation of Russia and the USA.

\section{References}

Alexander, S. G., \& Mészáros, P. 1991, ApJ, 372, 565

Araya, R. A., \& Harding, A. K. 1996, ApJ, 463, 33

Araya, R. A., \& Harding, A. K. 1999, ApJ, 517, 334

Araya-Góchez, R. A., \& Harding, A. K. 2000, ApJ, 544, 1067

Arnaud, K. A. 1996, in Astronomical Data Analysis Software and Systems V,

ed. G. H. Jacoby, \& J. Barnes (San Francisco: ASP), ASP Conf. Ser., 101, 17 Arons, J. 1993, ApJ, 408, 160

Basko, M. M., \& Sunyaev, R. A. 1976, MNRAS, 175, 395

Becker, P. A. 1998, ApJ, 498, 790

Becker, P. A., \& Wolff, M. T. 2005, ApJ, 630, 465

Becker, P. A., \& Wolff, M. T. 2007, ApJ, 654, 435

Blandford, R. D., Applegate, J. H., \& Hernquist, L. 1983, MNRAS, 204, 1025

Bonazzola, S., Heyvaerts, J., \& Puget, J. L. 1979, A\&A, 78, 53

Bulik, T., Mészáros, P., Woo, J. W., Hagase, F., \& Makishima, K. 1992, ApJ, 395,564

Bulik, T., Riffert, H., Mészáros, P., et al. 1995, ApJ, 444, 405

Caballero, I., Kretschmar, P., Santangelo, A., et al. 2007, A\&A, 465, L21

Canuto, V., \& Ventura, J. 1977, Fund. Cosm. Phys., 2, 203

Coburn, W., Heindl, W. A., Rothschild, R. E., et al. 2002, ApJ, 580, 394

Coburn, W., Kretschmar, P., Kreykenbohm, I., et al. 2005, Multiple Cyclotron

Lines in V0332+53, ATEL, 381
Daugherty, J. K., \& Harding, A. K. 1986, ApJ, 309, 362

Freeman, P. E., Lamb, D. Q., Wang, J. C. L., et al. 1999, ApJ, 524, 772

Ghosh, P., \& Lamb, F. K. 1978, ApJ, 223, L83

Gil, J. A., Melikidze, G. I., \& Mitra, D. 2002, A\&A, 388, 235

Grove, J. E., Strickman, M. S., Johnson, W. N., et al. 1995, ApJ, 438, L25

Harding, A. K., \& Lai, D. 2006, Rep. Prog. Phys, 69, 2631

Harding, A. K., Kirk, J. G., Galloway, D. J., \& Mészáros, P. 1984, ApJ, 278, 369

Heindl, W. A., Coburn, W., Gruber, D. E., et al. 2000, in Proc. 5th Compton

Symp., ed. M. L. McConnell, \& J. M. Ryan (New York: AIP), AIP Conf. Proc., 510, 1230

Heindl, W. A., Coburn, W., Gruber, D. E., et al. 1999, ApJ, 521, L49

Heindl, W. A., Rothschild, R. E., Coburn, W., et al. 2004, in X-ray Timing 2003: Rossi and Beyond, ed. P. Kaaret, F. K. Lamb, \& J. H. Swank (New York: AIP), AIP Conf. Proc., 714, 323

Houck, J. C., \& Denicola, L. A. 2000, in Astronomical Data Analysis Software and Systems IX, ed. N. Manset, C. Veillet, \& D. Crabtree (San Francisco: ASP), ASP Conf. Ser., 216, 591

Inoue, H., Kunieda, H., White, N., et al. 2005, Suzaku detection of cyclotron line near $50 \mathrm{keV}$ for A0535+26, ATEL, 613

Isenberg, M., Lamb, D. Q., \& Wang, J. C. L. 1998a, ApJ, 493, 154

Isenberg, M., Lamb, D. Q., \& Wang, J. C. L. 1998b, ApJ, 505, 688

Kendziorra, E., Kretschmar, P., Pan, H. C., et al. 1994, A\&A, 291, 31

Kretschmar, P., Kreykenbohm, I., Pottschmidt, K., et al. 2005, Integral observes possible cyclotron line at $47 \mathrm{keV}$ for $1 \mathrm{~A} 0535+262$, ATEL, 601

Kreykenbohm, I., Kretschmar, P., Wilms, J., et al. 1999, A\&A, 341, 141

Kreykenbohm, I., Coburn, W., Wilms, J., et al. 2002, A\&A, 395, 129

Kreykenbohm, I., Mowlavi, N., Produit, N., et al. 2005, A\&A, 433, 45

La Barbera, A., Segreto, A., Santangelo, A., Kreykenbohm, I., \& Orlandini, M. 2005, A\&A, 438, 617

Lamb, D. Q., Wang, J. C. L., \& Wasserman, I. M. 1990, ApJ, 363, 670

Lamb, F. K., Pethick, C. J., \& Pines, D. 1973, ApJ, 184, 271

Latal, H. G. 1986, ApJ, 309, 372

Makishima, K., Mihara, T., Ishida, M., et al. 1990a, ApJ, 365, L59

Makishima, K., Ohashi, T., Kawai, N., et al. 1990b, PASJ, 42, 295

Makishima, K., Mihara, T., Nagase, F., \& Tanaka, Y. 1999, ApJ, 525, 978

Mészáros, P. 1992, High-Energy Radiation from Magnetized Neutron Stars (Chicago: University of Chicago Press)

Mészáros, P., \& Nagel, W. 1985, ApJ, 298, 147

Mészáros, P., Harding, A. K., Kirk, J. G., \& Galloway, D. J. 1983, ApJ, 266, L33

Mihara, T. 1995, Ph.D. Thesis, RIKEN, Tokyo

Mihara, T., Makishima, K., Ohashi, T., Sakao, T., \& Tashiro, M. 1990, Nature, 346,250

Mowlavi, N., Kreykenbohm, I., Shaw, S. E., et al. 2006, A\&A, 451, 187

Nagel, W. 1980, ApJ, 236, 904

Nagel, W. 1981, ApJ, 251, 288

Nakajima, M., Mihara, T., Makishima, K., \& Niko, H. 2006, ApJ, 646, 1125

Nishimura, O. 2003, PASJ, 55, 849

Nishimura, O. 2005, PASJ, 57, 769

Orlandini, M., \& Fiume, D. D. 2001, AIP Conf. Proc., 599, 283

Ostriker, J. P., \& Davidson, K. 1973, in IAU Symp., ed. H. Bradt, \& R. Giacconi, 55,143

Pottschmidt, K., Kreykenbohm, I., Wilms, J., et al. 2005, ApJ, 634, L97

Pravdo, S. H., \& Bussard, R. W. 1981, ApJ, 246, L115

Santangelo, A., Segreto, A., Del Sordo, S., et al. 2000, BAAS, 32, 1230

Santangelo, A., Segreto, A., Giarrusso, S., et al. 1999, ApJ, 523, L85

Sina, R. 1996, Ph.D. Thesis, University of Maryland

Slater, G., Salpeter, E. E., \& Wasserman, I. 1982, ApJ, 255, 293

Staubert, R. 2003, ChJAAS, 3, 270

Staubert, R., Shakura, N. I., Postnov, K., et al. 2007, A\&A, 465, L25

Tanaka, Y. 1983, IAU Circ. 3891, 2

Tanaka, Y. 1986, Observations of Compact X-Ray Sources, ed. D. Mihalas, \&

K.-H. A. Winkler, Lecture Notes in Physics (Berlin: Springer), 255, 198

Terada, Y., Mihara, T., Nakajima, M., et al. 2006, ApJ, 648, L139

Trümper, J., Pietsch, W., Reppin, C., et al. 1977, NY Acad. Sc., 302, 538

Trümper, J., Pietsch, W., Reppin, C., et al. 1978, ApJ, 219, L105

Tsygankov, S. S., Lutovinov, A. A., Churazov, E. M., \& Sunyaev, R. A. 2006,

MNRAS, 371, 19

Urpin, V. A., Levshakov, S. A., \& Iakovlev, D. G. 1986, MNRAS, 219, 703

Wang, J. C. L., Wasserman, I. M., \& Salpeter, E. E. 1988, ApJ, 68, S735

Wang, J. C. L., Wasserman, I. M., \& Salpeter, E. E. 1989a, ApJ, 338, 343

Wang, J. C. L., Lamb, D. Q., Loredo, T. J., Wasserman, I. M., \& Salpeter, E. E. 1989b, Phys. Rev. Lett., 63, 1550

Wilson, C. A., \& Finger, M. H. 2005, RXTE confirms cyclotron line near $50 \mathrm{keV}$ for A $0535+26$, ATEL, 605

Yahel, R. Z. 1979, ApJ, 229, 73 